\title{
Fourth-Order Adjoint Sensitivity Analysis of an OECD/NEA Reactor Physics Benchmark: II. Mathematical Expressions and CPU-Time Comparisons for Computing $4^{\text {th }}$-Order Sensitivities
}

\author{
Dan Gabriel Cacuci*, Ruixian Fang \\ Center for Nuclear Science and Energy, Department of Mechanical Engineering, University of South Carolina, Columbia, SC, USA \\ Email: *cacuci@cec.sc.edu, fangr@cec.sc.edu
}

How to cite this paper: Cacuci, D.G. and Fang, R.X. (2021) Fourth-Order Adjoint Sensitivity Analysis of an OECD/NEA Reactor Physics Benchmark: II. Mathematical Expressions and CPU-Time Comparisons for Computing $4^{\text {th }}$-Order Sensitivities. American Journal of Computational Mathematics, 11, 133-156.

https://doi.org/10.4236/ajcm.2021.112010

Received: April 2, 2021

Accepted: May 23, 2021

Published: May 26, 2021

Copyright $\odot 2021$ by author(s) and Scientific Research Publishing Inc. This work is licensed under the Creative Commons Attribution International License (CC BY 4.0).

http://creativecommons.org/licenses/by/4.0/

(c) (i) Open Access

\begin{abstract}
This work extends to fourth-order previously published work on developing the adjoint sensitivity and uncertainty analysis of the numerical model of a polyethylene-reflected plutonium (acronym: PERP) OECD/NEA reactor physics benchmark. Previous works showed that the third-order sensitivities of the PERP leakage response with respect to these total microscopic cross sections are far larger than the corresponding $1^{\text {st }}$-order and $2^{\text {nd }}$-order ones, thereby having the largest impact on the uncertainties induced in the PERP benchmark's response. This finding has motivated the development of the original $4^{\text {th }}$-order formulas presented in this work, which are valid not only for the PERP benchmark but can also be used for computing the $4^{\text {th }}$-order sensitivities of response of any nuclear system involving fissionable material and internal or external neutron sources. Subsequent works will use the adjoint-based mathematical expressions obtained in this work to compute exactly and efficiently the numerical values of the largest fourth-order sensitivities of the PERP benchmark's response to the total microscopic cross sections, and use them for a pioneering fourth-order uncertainty analysis of the PERP benchmark's response.
\end{abstract}

\section{Keywords}

Polyethylene-Reflected Plutonium Sphere, $4^{\text {th }}$-Order Adjoint Sensitivity

Analysis, Microscopic Total Cross Sections

\section{Introduction}

By applying the $4^{\text {th }}$-Order Comprehensive Adjoint Sensitivity Analysis Metho- 
dology $\left(4^{\text {th }}-\right.$ CASAM) for linear system [1], this work extends the results reported in the accompanying Part I [2] by presenting the derivation of the expressions of the $4^{\text {th }}$-order sensitivities of the leakage response of the polyethylene-reflected plutonium (acronym: PERP) OECD/NEA reactor physics benchmark [3] to the PERP benchmark's 180 group-averaged total microscopic cross sections. The companion work [4] will present numerical results for the $4^{\text {th }}$-order sensitivities along with their impact on the uncertainties they induce in the PERP leakage response, and compare with the contributions stemming from the corresponding $1^{\text {st }}-, 2^{\text {nd }}$ - and $3^{\text {rd }}$-order sensitivities, which have been computed and reported in [5] [6].

This work is organized as follows: Section 2 presents the methodology for deriving the analytical expressions of the exact $4^{\text {th }}$-order sensitivities of the PERP benchmark's leakage response with respect to the total microscopic cross sections. This Section also presents comparisons of the CPU-times which would be required for computing $4^{\text {th }}$-order sensitivities using the finite-difference formulas-which would provide just approximate values for various sensitivities-or the forward sensitivity analysis methodology - which could provide exact values for the sensitivities of interest. It is shown that the $4^{\text {th }}$-CASAM used in this work is by far more efficient than any other method, while showing that the methodology is exact, introducing no intrinsic methodological errors in the computation of sensitivities. Section 3 concludes this work.

\section{Analytical Expressions of the Exact Fourth-Order Sensitivities of the PERP Leakage Response to Total Microscopic Cross Sections}

This section continuing the work presented in Part I [2] presents the derivation of the exact analytical expressions of the $4^{\text {th }}$-order sensitivities of the leakage response of the polyethylene-reflected plutonium (acronym: PERP) metal sphere OECD/NEA benchmark [3] with respect to this benchmark's total microscopic cross sections.

This Section presents the three fundamental deterministic (as opposed to "statistical") methods for computing the response sensitivities, namely: 1) finite differences; 2) the forward sensitivity analysis method; and 3) the Fourth-Order Comprehensive Adjoint Sensitivity Analysis Methodology (4 $4^{\text {th }}-$ CASAM) for linear systems, conceived and developed by Cacuci [1]. The main features of applying these methods for the deterministic computation of the 4th-order sensitivities of the PERP benchmark leakage response with respect to the group-averaged microscopic total cross sections are presented in Section 2.2.

\subsection{Mathematical/Computational Modeling of the PERP Benchmark}

The dimensions and material composition of the spherical polyethylene-reflected plutonium (acronym: PERP) benchmark considered in this work are presented in Table A1 in the Appendix. The quantity of interest in this work, which will 
be called the "response," is the leakage of neutrons out of the PERP sphere, which has been measured experimentally [3]. The numerical modeling of the neutron flux distribution within the PERP benchmark, as well as the computation of the PERP leakage response has been performed using the multigroup discrete ordinates particle transport code PARTISN [7], together with neutron sources computed using the code SOURCES4C [8] and the MENDF71X [9] 618-group cross section data collapsed to $G=30$ energy groups. The neutron flux distribution within the PERP benchmark, as well as the leakage of neutrons through the outer boundary of the PERP benchmark has been modeled using the standard multigroup form of the Boltzmann neutron transport equation subject to the boundary condition of no incoming flux, with an internal spontaneous fission source, which can be written in the following form:

$$
\begin{gathered}
B^{g}(\boldsymbol{\alpha}) \varphi^{g}(r, \boldsymbol{\Omega})=Q^{g}(\boldsymbol{\alpha}), \quad g=1, \cdots, G, \\
\varphi^{g}(r, \boldsymbol{\Omega})=0, r=r_{d}, \boldsymbol{\Omega} \cdot \boldsymbol{n}<0, \quad g=1, \cdots, G,
\end{gathered}
$$

where $r_{d}$ is the external radius of the PERP sphere, and where the operator $B^{g}(\alpha)$ and the spontaneous source $Q^{g}(\alpha)$ are defined as follows,

$$
\begin{aligned}
& B^{g}(\boldsymbol{\alpha}) \varphi^{g}(r, \boldsymbol{\Omega}) \triangleq \boldsymbol{\Omega} \cdot \nabla \varphi^{g}(r, \boldsymbol{\Omega})+\Sigma_{t}^{g}(r) \varphi^{g}(r, \boldsymbol{\Omega}) \\
& -\sum_{h=1}^{G} \int_{4 \pi} \mathrm{d} \boldsymbol{\Omega}^{\prime} \varphi^{h}\left(r, \boldsymbol{\Omega}^{\prime}\right)\left[\sum_{s}^{h \rightarrow g}\left(\boldsymbol{\alpha} ; r, \boldsymbol{\Omega}^{\prime} \rightarrow \boldsymbol{\Omega}\right)+\chi^{g}(\boldsymbol{\alpha} ; r)\left(v \Sigma_{f}\right)^{h}(\boldsymbol{\alpha} ; r)\right], \\
& \mathbf{Q}^{g}(\boldsymbol{\alpha}) \triangleq \sum_{k=1}^{N_{f}} \lambda_{k} N_{k} F_{k}^{S F} v_{k}^{S F}\left(\frac{2}{\sqrt{\pi a_{k}^{3} b_{k}}} \mathrm{e}^{-\frac{a_{k} b_{k}}{4}}\right) \int_{E^{g+1}}^{E^{g}} \mathrm{~d} E \mathrm{e}^{-E / a_{k}} \sinh \sqrt{b_{k} E},
\end{aligned}
$$

where $N_{f}$ denotes the total number of spontaneous-fission isotopes. For an actinide nuclide $k$, the spontaneous source depends on the following parameters: 1) the decay constant $\lambda_{k} ; 2$ ) the atom density $N_{k} ; 3$ ) the average number of neutrons per spontaneous fission $\left.v_{k}^{S F} ; 4\right)$ the spontaneous fission branching ratio $F_{k}^{S F}$; and 5) the evaluated parameters $a_{k}$ and $b_{k}$, which are used in a Watt's fission spectrum to approximate the spontaneous fission neutron spectrum. The PERP benchmark has $N_{f}=2$ spontaneous-fission isotopes, namely "isotope 1 " $\left.{ }^{239} \mathrm{Pu}\right)$ and "isotope 2 " $\left({ }^{240} \mathrm{Pu}\right)$. Thus, the index $k=1$ refers to "isotope 1 " ${ }^{239} \mathrm{Pu}$ ) while $k=2$ refers to "isotope 2 " $\left.{ }^{240} \mathrm{Pu}\right)$. The vector $\alpha$, which appears in the arguments in Equation (1), is defined as follows:

$$
\begin{aligned}
& \boldsymbol{\alpha} \triangleq\left[\alpha_{1}, \cdots, \alpha_{\boldsymbol{T P}}\right]^{\dagger} \triangleq\left[\boldsymbol{q} ; \boldsymbol{N} ; \boldsymbol{\sigma}_{s} ; \boldsymbol{\sigma}_{t} ; \boldsymbol{\sigma}_{f} ; \boldsymbol{v} ; \boldsymbol{p}\right]^{\dagger}, \\
& \text { where } \boldsymbol{T P} \triangleq \boldsymbol{J} \boldsymbol{Q}+\boldsymbol{I}+\boldsymbol{J S} \boldsymbol{X}+\boldsymbol{J T T} \boldsymbol{X}+\boldsymbol{J F X}+\boldsymbol{J N U}+\boldsymbol{J} \chi .
\end{aligned}
$$

The components of the TP-dimensional vector $\alpha$ are the uncertain model and response parameters, and are described in the Appendix. In Equation (5) and throughout this work, the dagger will be used to denote "transposition." The nominal values of the parameters in Equation (5), as well as of all other quantities in this work, will be denoted using the superscript "zero," e.g., $\boldsymbol{\alpha}^{0} \triangleq\left[\alpha_{1}^{0}, \cdots, \alpha_{T P}^{0}\right]^{\dagger}$. The meanings of other quantities appearing in Equations (1) through (4) are described in the Appendix and in the Nomenclature. 
The mathematical expression of the PERP benchmark's leakage response, denoted as $L(\alpha)$, is provided below:

$$
L(\boldsymbol{\alpha}) \triangleq \int_{S_{b}} \mathrm{~d} S \sum_{g=1}^{G} \int_{\boldsymbol{\Omega} \cdot \boldsymbol{n}>0} \mathrm{~d} \boldsymbol{\Omega} \boldsymbol{\Omega} \cdot \boldsymbol{n} \varphi^{g}(r, \boldsymbol{\Omega}),
$$

where $S_{b}$ is the external surface area of the PERP sphere.

For the mathematical derivations to follow in this work, it will be convenient to use the matrix-form of Equations (1) and (2), which is as follows:

$$
\begin{gathered}
\boldsymbol{B}(\alpha) \varphi(r, \boldsymbol{\Omega})=\boldsymbol{Q}(\alpha), \\
\varphi(r, \boldsymbol{\Omega})=\mathbf{0}, \quad r=r_{d}, \boldsymbol{\Omega} \cdot \boldsymbol{n}<0,
\end{gathered}
$$

where

$\boldsymbol{B}(\alpha) \triangleq\left(\begin{array}{ccccc}B_{11}(\alpha) & \bullet & B_{1 g}(\alpha) & \cdot & B_{1 G}(\alpha) \\ \bullet & \bullet & \bullet & \bullet & \bullet \\ B_{g 1}(\alpha) & \bullet & B_{g g}(\alpha) & \bullet & B_{g G}(\alpha) \\ \bullet & \bullet & \bullet & \bullet & \bullet \\ B_{G 1}(\alpha) & \bullet & B_{G g}(\alpha) & \bullet & B_{G G}(\alpha)\end{array}\right) ; \varphi(r, \Omega) \triangleq\left(\begin{array}{c}\varphi^{1} \\ \cdot \\ \varphi^{g} \\ \cdot \\ \varphi^{G}\end{array}\right) ; \boldsymbol{Q}(\alpha) \triangleq\left(\begin{array}{c}Q^{1} \\ \cdot \\ Q^{g} \\ \cdot \\ Q^{G}\end{array}\right)$

with components defined below:

$$
\begin{aligned}
& B_{g h}(\boldsymbol{\alpha}) \triangleq \delta_{g, h} B_{g g}^{0}(\boldsymbol{\alpha})-B_{g h}^{1}(\boldsymbol{\alpha}) ; g, h=1, \cdots, G ; \\
& B_{g g}^{0}(\boldsymbol{\alpha}) \varphi^{g}(r, \boldsymbol{\Omega}) \triangleq\left[\boldsymbol{\Omega} \cdot \nabla+\Sigma_{t}^{g}(\boldsymbol{\alpha} ; r)\right] \varphi^{g}(r, \boldsymbol{\Omega}) ; \\
& B_{g h}^{1}(\boldsymbol{\alpha}) \varphi^{h}\left(r, \boldsymbol{\Omega}^{\prime}\right) \triangleq \int_{4 \pi} \mathrm{d} \boldsymbol{\Omega}^{\prime}\left[\Sigma_{s}^{h \rightarrow g}\left(\boldsymbol{\alpha} ; r, \boldsymbol{\Omega}^{\prime} \rightarrow \boldsymbol{\Omega}\right)+\chi^{g}(\boldsymbol{\alpha} ; r)\left(v \Sigma_{f}\right)^{h}(\boldsymbol{\alpha} ; r)\right] .
\end{aligned}
$$

In Equation (10), the notation $\delta_{g, h}$ denotes the Kronecker delta-functional, which is defined as usual, i.e., $\delta_{g, h}=1$, if $g=h$ and $\delta_{g, h}=0$, if $g \neq h$.

$$
\begin{aligned}
& \text { 2.2. Computation of } \partial^{4} L(\alpha) / \partial t_{j 1} \partial t_{j 2} \partial t_{j 3} \partial t_{j 4}, j 1=1, \cdots, J T X ; \\
& j 2=1, \cdots, j 1 ; j 3=1, \cdots, j 2 ; j 4=1, \cdots, j 3
\end{aligned}
$$

Since this work aims at applying the $4^{\text {th }}$-Order Comprehensive Sensitivity Analysis Methodology ( $4^{\text {th }}-$ CASAM) to obtain the closed form expressions of the $4^{\text {th }}$-order sensitivities of the PERP leakage response with respect to the groupaveraged total microscopic cross sections, the proliferation of indices, superscripts and subscripts is unavoidable. Nevertheless, "subscripted-subscripts" can be avoided by using subscripts of the form $j 1=1, \cdots, J T X ; j 2=1, \cdots, j 1$, where the index $j 1$ will be used to index the $1^{\text {st }}$-order sensitivities, and where the index $j 2$ will be used (in addition to $j 1$ ) to index the $2^{\text {nd }}$-order sensitivities. Furthermore, the index $j 3$ will be used (in addition to $j 1$ and $j$ ) to index the $3^{\text {rd }}$-order sensitivities; the index $j 4$ will be used (in addition to $j 1, j 2$ and $j 3$ ) to index the $4^{\text {th }}$-order sensitivities. As described in the Appendix, JTX $=I \times G=180$ represents the total number of components $\sigma_{t, i}^{g}$ in the vector

$$
\begin{aligned}
& \boldsymbol{t} \triangleq\left[t_{1}, \cdots, t_{J T X}\right]^{\dagger} \triangleq\left[\sigma_{t, i=1}^{1}, \sigma_{t, i=1}^{2}, \cdots, \sigma_{t, i=1}^{G}, \cdots, \sigma_{t, i}^{g}, \cdots, \sigma_{t, i=I}^{1}, \cdots, \sigma_{t, i=I}^{G}\right]^{\dagger}, \text { for } \\
& i=1, \cdots, I=6 ; g=1, \cdots, G=30 .
\end{aligned}
$$


Finite-difference formulas that can be used to compute approximately the fourth-order response sensitivities with respect to the model parameters will be discussed in Subsection 2.2.1. The Forward Sensitivity Analysis Methodology will be discussed in Subsection 2.2.2. The $4^{\text {th }}$-CASAM will be discussed in Subsection 2.2.3, while Subsection 2.2.4 will present a comparative discussion of the computational resources and times (CPU) which are required by these three deterministic methods.

\subsubsection{Re-Computations with Finite-Difference Approximation}

The $4^{\text {th }}$-order unmixed sensitivities, $\partial^{4} L(\alpha) / \partial \alpha_{j}^{4}$, of the leakage response, $L(\boldsymbol{\alpha})$, with respect to all the model parameters, can be approximately computed by re-computations using the well-known finite-difference formula presented below:

$$
\frac{\partial^{4} L(\alpha)}{\left(\partial \alpha_{j}\right)^{4}} \approx \frac{1}{h_{j}^{4}}\left(L_{j+2}-4 L_{j+1}+6 L_{j}-4 L_{j-1}+L_{j-2}\right)+\mathrm{O}\left(h_{j}^{2}\right), j=1, \cdots, T P,
$$

where $L_{j+2} \triangleq L\left(\alpha_{j}+2 h_{j}\right), \quad L_{j+1} \triangleq L\left(\alpha_{j}+h_{j}\right), \quad L_{j-1} \triangleq L\left(\alpha_{j}-h_{j}\right)$, $L_{j-2} \triangleq L\left(\alpha_{j}-2 h_{j}\right)$ and $h_{j}$ denotes a "judiciously-chosen" variation in the parameter $\alpha_{j}$ around its nominal value $\alpha_{j}^{0}$. The $4^{\text {th }}$-order mixed sensitivities, $\partial^{4} L(\alpha) / \partial \alpha_{j 1} \partial \alpha_{j 2} \partial \alpha_{j 3} \partial \alpha_{j 4}$, can be calculated by using the following finite-difference formula:

$$
\begin{aligned}
& \frac{\partial^{4} L(\alpha)}{\partial \alpha_{j 1} \partial \alpha_{j 2} \partial \alpha_{j 3} \partial \alpha_{j 4}} \approx \frac{1}{16 h_{j 1} h_{j 2} h_{j 3} h_{j 4}}\left(L_{j 1+1, j 2+1, j 3+1, j 4+1}-L_{j 1+1, j 2+1, j 3+1, j 4-1}\right. \\
& -L_{j 1+1, j 2+1, j 3-1, j 4+1}+L_{j 1+1, j 2+1, j 3-1, j 4-1}-L_{j 1+1, j 2-1, j 3+1, j 4+1}+L_{j 1+1, j 2-1, j 3+1, j 4-1} \\
& +L_{j 1+1, j 2-1, j 3-1, j 4+1}-L_{j 1+1, j 2-1, j 3-1, j 4-1}-L_{j 1-1, j 2+1, j 3+1, j 4+1}+L_{j 1-1, j 2+1, j 3+1, j 4-1} \\
& +L_{j 1-1, j 2+1, j 3-1, j 4+1}-L_{j 1-1, j 2+1, j 3-1, j 4-1}+L_{j 1-1, j 2-1, j 3+1, j 4+1}-L_{j 1-1, j 2-1, j 3+1, j 4-1} \\
& \left.-L_{j 1-1, j 2-1, j 3-1, j 4+1}+L_{j 1-1, j 2-1, j 3-1, j 4-1}\right)+\mathrm{O}\left(h_{j 1}^{2}, h_{j 2}^{2}, h_{j 3}^{2}, h_{j 4}^{2}\right)
\end{aligned}
$$

where $L_{j 1+1, j 2+1, j 3+1, j 4+1} \triangleq L\left(\alpha_{j 1}+h_{j 1}, \alpha_{j 2}+h_{j 2}, \alpha_{j 3}+h_{j 3}, \alpha_{j 4}+h_{j 4}\right)$, etc. The finite difference formulas introduce their intrinsic "methodological errors" of order $\mathrm{O}\left(h_{j 1}^{2}, h_{j 2}^{2}, h_{j 3}^{2}, h_{j 4}^{2}\right)$ which are in addition to, and independent of, the errors that might be incurred in the computation of $\partial^{4} L(\alpha) / \partial \alpha_{j 1} \partial \alpha_{j 2} \partial \alpha_{j 3} \partial \alpha_{j 4}$.

\subsubsection{Forward Sensitivity Analysis Methodology (FSAM)}

The fourth-order partial sensitivity of $L(\boldsymbol{\alpha})$ to four generic model parameters, $\alpha_{j 1}, \alpha_{j 2}, \alpha_{j 3}, \alpha_{j 4}$, has the following expression:

$$
\begin{gathered}
\frac{\partial^{4} L(\boldsymbol{\alpha})}{\partial \alpha_{j 4} \partial \alpha_{j 3} \partial \alpha_{j 2} \partial \alpha_{j 1}} \triangleq \int_{S_{b}} \mathrm{~d} S \sum_{g=1}^{G} \int_{\boldsymbol{\Omega} \cdot \boldsymbol{n}>0} \mathrm{~d} \boldsymbol{\Omega} \boldsymbol{\Omega} \cdot \boldsymbol{n} \frac{\partial^{4} \varphi^{g}(r, \boldsymbol{\Omega})}{\partial \alpha_{j 4} \partial \alpha_{j 3} \partial \alpha_{j 2} \partial \alpha_{j 1}}, \\
j 1=1, \cdots, T P ; j 2=1, \cdots, j 1 ; j 3=1, \cdots, j 2, \quad j 4=1, \cdots, j 3 .
\end{gathered}
$$

In turn, the fourth-order derivative $\partial^{4} \varphi^{g}(r, \Omega) / \partial \alpha_{j 1} \partial \alpha_{j 2} \partial \alpha_{j 3} \partial \alpha_{j 4}$ is the solution of the fourth-order G-derivative of Equations (1) and (2) with respect to $\alpha_{j 1}, \alpha_{j 2}, \alpha_{j 3}, \alpha_{j 4}$, namely: 


$$
\begin{aligned}
& B^{g}(\alpha)\left[\frac{\partial^{4} \varphi^{g}(r, \boldsymbol{\Omega})}{\partial \alpha_{j 4} \partial \alpha_{j 3} \partial \alpha_{j 2} \partial \alpha_{j 1}}\right]=\frac{\partial^{4} S^{g}\left[\varphi^{g}(r, \boldsymbol{\Omega}), r, \boldsymbol{\Omega}\right]}{\partial \alpha_{j 4} \partial \alpha_{j 3} \partial \alpha_{j 2} \partial \alpha_{j 1}}, \\
& g=1, \cdots, G ; j 1=1, \cdots, T P ; j 2=1, \cdots, j 1 ; j 3=1, \cdots, j 2 ; j 4=1, \cdots, j 3 ; \\
& \frac{\partial^{4} \varphi^{g}(r, \boldsymbol{\Omega})}{\partial \alpha_{j 4} \partial \alpha_{j 3} \partial \alpha_{j 2} \partial \alpha_{j 1}}=0, \boldsymbol{\Omega} \cdot \boldsymbol{n}<0, \\
& g=1, \cdots, G ; j 1=1, \cdots, T P ; j 2=1, \cdots, j 1 ; j 3=1, \cdots, j 2 ; j 4=1, \cdots, j 3 ;
\end{aligned}
$$

where

$$
\frac{\partial^{4} S^{g}\left[\varphi^{g}(r, \boldsymbol{\Omega}), r, \boldsymbol{\Omega}\right]}{\partial \alpha_{j 4} \partial \alpha_{j 3} \partial \alpha_{j 2} \partial \alpha_{j 1}}=\frac{\partial}{\partial \alpha_{j 4}}\left\{\frac{\partial^{3} S^{g}\left[\varphi^{g}(r, \boldsymbol{\Omega}), r, \boldsymbol{\Omega}\right]}{\partial \alpha_{j 3} \partial \alpha_{j 2} \partial \alpha_{j 1}}\right\} .
$$

Note that a "place-holder," denoted as [], has been explicitly used on the leftside of Equation (14) to indicate that the operator $B^{g}(\alpha)$ acts (linearly) on the function that appears in this place-holder. Evidently, the computations of all of the fourth-order sensitivities $\partial^{4} L(\alpha) / \partial \alpha_{j 4} \partial \alpha_{j 3} \partial \alpha_{j 2} \partial \alpha_{j 1}$ require $T P(T P+1)(T P+2)(T P+3) / 4$ ! large-scale computations to solve Equations (14) and (15), followed by $T P(T P+1)(T P+2)(T P+3) / 4$ ! small-scale computations for performing the integration represented by Equation (13).

\subsubsection{Fourth-Order Adjoint Sensitivity Analysis Methodology (4th-CASAM)}

In contradistinction to the FSAM, the 4th-CASAM does not use Equation (13) for computing 4th-order response sensitivities but instead uses the total G-differential of the expression that resulted from the application of the 3rd-CASAM, namely Equation (91) or, equivalently, Equation (92) from Ref. [2], which is reproduced below, for convenience:

$$
\begin{aligned}
& \left\{\frac{\partial^{3} L\left(\boldsymbol{\alpha}, \boldsymbol{\varphi} ; \boldsymbol{\psi}^{(1)} ; \boldsymbol{\psi}^{(2)} ; \boldsymbol{\psi}^{(3)}\right)}{\partial t_{j 3} \partial t_{j 2} \partial t_{j 1}}\right\}_{\boldsymbol{\alpha}^{0}} \\
& =-\left\{\left\langle\psi_{1}^{(3)}(j 2 ; j 1 ; r, \boldsymbol{\Omega}), \boldsymbol{S}(j 3 ; \boldsymbol{\alpha}) \boldsymbol{\varphi}(r, \boldsymbol{\Omega})\right\rangle_{(1)}\right\}_{\alpha^{0}} \\
& -\left\{\left\langle\boldsymbol{\psi}_{2}^{(3)}(j 2 ; j 1 ; r, \boldsymbol{\Omega}), \boldsymbol{S}(j 3 ; \boldsymbol{\alpha}) \boldsymbol{\psi}^{(1)}(r, \boldsymbol{\Omega})\right\rangle_{(1)}\right\}_{\alpha^{0}} \\
& -\left\{\left\langle\boldsymbol{\psi}_{3}^{(3)}(j 2 ; j 1 ; r, \boldsymbol{\Omega}), \boldsymbol{S}(j 3 ; \boldsymbol{\alpha}) \boldsymbol{\psi}_{1}^{(2)}(j 1 ; r, \boldsymbol{\Omega})\right\rangle_{(1)}\right\}_{\boldsymbol{\alpha}^{0}} \\
& -\left\{\left\langle\boldsymbol{\psi}_{4}^{(3)}(j 2 ; j 1 ; r, \boldsymbol{\Omega}), \boldsymbol{S}(j 3 ; \boldsymbol{\alpha}) \boldsymbol{\psi}_{2}^{(2)}(j 1 ; r, \boldsymbol{\Omega})\right\rangle_{(1)}\right\}_{\boldsymbol{\alpha}^{0}},
\end{aligned}
$$

for $j 1=1, \cdots, J T X ; j 2=1, \cdots, j 1 ; j 3=1, \cdots, j 2$.

The $1^{\text {st }}$, $2^{\text {nd }}$ - and $3^{\text {rd }}$-level adjoint functions $\psi^{(1)}, \psi^{(2)}$ and $\psi^{(3)}$, which appear in Equation (17) are the solutions of the following systems which have been derived in [2]:

1) The $1^{\text {st }}$-level adjoint function $\psi^{(1)}$ is the solution of the $1^{\text {st }}$-Level Adjoint Sensitivity System ( $1^{\text {st }}$-LASS), which is defined as follows:

$$
\boldsymbol{A}(\boldsymbol{\alpha}) \boldsymbol{\psi}^{(1)}(r, \boldsymbol{\Omega})=\boldsymbol{I}\left[\boldsymbol{\Omega} \cdot \boldsymbol{n} \delta\left(r-r_{d}\right)\right] ; \boldsymbol{I} \triangleq[1, \cdots, 1, \cdots, 1]^{\dagger} .
$$




$$
\boldsymbol{\psi}^{(1)}(r, \boldsymbol{\Omega})=\mathbf{0}, r=r_{d}, \boldsymbol{\Omega} \cdot \boldsymbol{n}>0,
$$

where $\boldsymbol{A}(\boldsymbol{\alpha})$ denotes the operator adjoint to $\boldsymbol{B}(\boldsymbol{\alpha})$, having components $A_{g h}(\alpha) \triangleq\left[B_{h g}(\alpha)\right]^{*}$, where the symbol [ ] indicates "formal adjoint operator."

2) The $2^{\text {nd }}-$ level adjoint function

$\boldsymbol{\psi}^{(2)}(j 1 ; r, \boldsymbol{\Omega}) \triangleq\left[\boldsymbol{\psi}_{1}^{(2)}(j 1 ; r, \boldsymbol{\Omega}), \boldsymbol{\psi}_{2}^{(2)}(j 1 ; r, \boldsymbol{\Omega})\right]^{\dagger}$ is the solution of the $2^{\text {nd }}$-Level Adjoint Sensitivity System ( $2^{\text {nd }}-$ LASS), which is defined as follows:

$$
\begin{gathered}
\left\{\boldsymbol{A}(\boldsymbol{\alpha}) \boldsymbol{\psi}_{1}^{(2)}(j 1 ; r, \boldsymbol{\Omega})\right\}_{\alpha^{0}}=-\left\{\boldsymbol{S}(j 1 ; \boldsymbol{\alpha}) \boldsymbol{\psi}^{(1)}(r, \boldsymbol{\Omega})\right\}_{\boldsymbol{\alpha}^{0}}, j 1=1, \cdots, J T X, \\
\left\{\boldsymbol{B}(\boldsymbol{\alpha}) \boldsymbol{\psi}_{2}^{(2)}(j 1 ; r, \boldsymbol{\Omega})\right\}_{\alpha^{0}}=-\{\boldsymbol{S}(j 1 ; \boldsymbol{\alpha}) \boldsymbol{\varphi}(r, \boldsymbol{\Omega})\}_{\alpha^{0}} ; j 1=1, \cdots, J T X, \\
\boldsymbol{\psi}_{1}^{(2)}(j 1 ; r, \boldsymbol{\Omega})=\mathbf{0}, r=r_{d}, \boldsymbol{\Omega} \cdot \boldsymbol{n}>0 ; j 1=1, \cdots, J T X, \\
\boldsymbol{\psi}_{2}^{(2)}(j 1 ; r, \boldsymbol{\Omega})=\mathbf{0}, \quad r=r_{d}, \boldsymbol{\Omega} \cdot \boldsymbol{n}<0 ; j 1=1, \cdots, J T X,
\end{gathered}
$$

where, for each $j 1=1, \cdots, J T X, \boldsymbol{S}(j 1 ; \boldsymbol{\alpha})$ is a $G \times G$ diagonal matrix having non-zero elements of the form $\partial \Sigma_{t}^{g}(\alpha) / \partial t_{j 1}, g=1, \cdots, G$ on its diagonal, i.e.,

$$
\boldsymbol{S}(j 1 ; \boldsymbol{\alpha}) \triangleq\left(\begin{array}{ccc}
\partial \Sigma_{t}^{1}(\boldsymbol{\alpha}) / \partial t_{j 1} & \cdot & 0 \\
\bullet & \bullet & \bullet \\
0 & \cdot & \partial \Sigma_{t}^{G}(\boldsymbol{\alpha}) / \partial t_{j 1}
\end{array}\right) .
$$

3) The $3^{\text {rd }}$-level adjoint function

$\boldsymbol{\psi}^{(3)}(j 2 ; j 1 ; r, \boldsymbol{\Omega}) \triangleq\left[\boldsymbol{\psi}_{1}^{(3)}(j 2 ; j 1 ; r, \boldsymbol{\Omega}), \cdots, \boldsymbol{\psi}_{4}^{(3)}(j 2 ; j 1 ; r, \boldsymbol{\Omega})\right]^{\dagger}$ is the solution of the $3^{\text {rd }}$-Level Adjoint Sensitivity System ( $3^{\text {rd }}$-LASS), which is defined as follows:

$$
\begin{aligned}
& \left\{\left(\begin{array}{cccc}
\boldsymbol{A}(\boldsymbol{\alpha}) & \mathbf{0} & \mathbf{0} & \boldsymbol{S}(j 1 ; \boldsymbol{\alpha}) \\
\mathbf{0} & \boldsymbol{B}(\boldsymbol{\alpha}) & \boldsymbol{S}(j 1 ; \boldsymbol{\alpha}) & \mathbf{0} \\
\mathbf{0} & \mathbf{0} & \boldsymbol{B}(\boldsymbol{\alpha}) & \mathbf{0} \\
\mathbf{0} & \mathbf{0} & \mathbf{0} & \boldsymbol{A}(\boldsymbol{\alpha})
\end{array}\right)\left(\begin{array}{l}
\boldsymbol{\psi}_{1}^{(3)}(j 2 ; j 1 ; r, \boldsymbol{\Omega}) \\
\boldsymbol{\psi}_{2}^{(3)}(j 2 ; j 1 ; r, \boldsymbol{\Omega}) \\
\boldsymbol{\psi}_{3}^{(3)}(j 2 ; j 1 ; r, \boldsymbol{\Omega}) \\
\boldsymbol{\psi}_{4}^{(3)}(j 2 ; j 1 ; r, \boldsymbol{\Omega})
\end{array}\right)\right\}_{\alpha^{0}} \\
& =-\left\{\left(\begin{array}{c}
\boldsymbol{S}(j 2 ; \boldsymbol{\alpha}) \boldsymbol{\psi}_{1}^{(2)}(j 1 ; r, \boldsymbol{\Omega}) \\
\boldsymbol{S}(j 2 ; \boldsymbol{\alpha}) \boldsymbol{\psi}_{2}^{(2)}(j 1 ; r, \boldsymbol{\Omega}) \\
\boldsymbol{S}(j 2 ; \boldsymbol{\alpha}) \boldsymbol{\varphi}(r, \boldsymbol{\Omega}) \\
\boldsymbol{S}(j 2 ; \boldsymbol{\alpha}) \boldsymbol{\psi}^{(1)}(r, \boldsymbol{\Omega})
\end{array}\right)\right\}_{\boldsymbol{\alpha}^{0}}, \text { for } j 1=1, \cdots, J T X ; j 2=1, \cdots, j 1,
\end{aligned}
$$

where $\boldsymbol{S}(j 2 ; \alpha)$ is a matrix having the same elements as $\boldsymbol{S}(j 1 ; \alpha)$, except that the index $j 1$ is replaced by the index $j$. The boundary conditions for the components of $\psi^{(3)}(j 2 ; j 1 ; r, \Omega)$ are as follows:

$$
\begin{aligned}
& \boldsymbol{\psi}_{i}^{(3)}(j 2 ; j 1 ; r, \boldsymbol{\Omega})=\mathbf{0}, r=r_{d} ; \boldsymbol{\Omega} \cdot \boldsymbol{n}<0 ; i=2,3 ; j 1=1, \cdots, J T X ; j 2=1, \cdots, j 1 ; \\
& \boldsymbol{\psi}_{i}^{(3)}(j 2 ; j 1 ; r, \boldsymbol{\Omega})=\mathbf{0}, r=r_{d} ; \boldsymbol{\Omega} \cdot \boldsymbol{n}>0 ; i=1,4 ; j 1=1, \cdots, J T X ; j 2=1, \cdots, j 1 .
\end{aligned}
$$

The $4^{\text {th }}$-order sensitivities of the PERP leakage response with respect to the group-averaged microscopic total cross sections are obtained after determining the total first-order G-differential of the expression of the $3^{\text {rd }}$-order sensitivities provided in Equation (17). By definition, this G-differential is obtained as follows: 


$$
\begin{aligned}
& \delta\left\{\frac{\partial^{3} L\left(\boldsymbol{\alpha}, \boldsymbol{\varphi} ; \boldsymbol{\psi}^{(1)} ; \boldsymbol{\psi}^{(2)} ; \boldsymbol{\psi}^{(3)}\right)}{\partial t_{j 3} \partial t_{j 2} \partial t_{j 1}}\right\}_{\boldsymbol{\alpha}^{0}} \\
& =\frac{\mathrm{d}}{\mathrm{d} \varepsilon}\left\{\frac{\partial^{3} L\left(\boldsymbol{\alpha}+\varepsilon \delta \boldsymbol{\alpha}, \boldsymbol{\varphi}+\varepsilon \delta \boldsymbol{\varphi} ; \boldsymbol{\psi}^{(1)}+\varepsilon \delta \boldsymbol{\psi}^{(1)} ; \boldsymbol{\psi}^{(2)}+\varepsilon \delta \boldsymbol{\psi}^{(2)} ; \boldsymbol{\psi}^{(3)}+\varepsilon \delta \boldsymbol{\psi}^{(3)}\right)}{\partial t_{j 3} \partial t_{j 2} \partial t_{j 1}}\right\}_{\alpha^{0}} \\
& =\delta\left\{\frac{\partial^{3} L\left(\boldsymbol{\alpha}, \boldsymbol{\varphi} ; \boldsymbol{\psi}^{(1)} ; \boldsymbol{\psi}^{(2)} ; \boldsymbol{\psi}^{(2)}\right)}{\partial t_{j 3} \partial t_{j 2} \partial t_{j 1}}\right\}_{d i r}+\delta\left\{\frac{\partial^{3} L\left(\boldsymbol{\alpha}, \boldsymbol{\varphi} ; \boldsymbol{\psi}^{(1)} ; \boldsymbol{\psi}^{(2)} ; \boldsymbol{\psi}^{(2)}\right)}{\partial t_{j 3} \partial t_{j 2} \partial t_{j 1}}\right\}_{\text {ind }} \\
& \quad \text { for } j 1=1, \cdots, J T X ; j 2=1, \cdots, j 1 ; j 3=1, \cdots, j 2,
\end{aligned}
$$

where the "direct-effect" term is defined as follows:

$$
\begin{aligned}
& \delta\left\{\frac{\partial^{3} L\left(\boldsymbol{\alpha}, \boldsymbol{\varphi} ; \boldsymbol{\psi}^{(1)} ; \boldsymbol{\psi}^{(2)} ; \boldsymbol{\psi}^{(3)}\right)}{\partial t_{j 3} \partial t_{j 2} \partial t_{j 1}}\right\}_{d i r} \\
\triangleq & -\left\{\left\langle\boldsymbol{\psi}_{1}^{(3)}(j 2 ; j 1 ; r, \boldsymbol{\Omega}),\left[\frac{\partial \boldsymbol{S}(j 3 ; \boldsymbol{\alpha})}{\partial \boldsymbol{\alpha}} \delta \boldsymbol{\alpha}\right] \boldsymbol{\varphi}(r, \boldsymbol{\Omega})\right\rangle_{(1)}\right\}_{\boldsymbol{\alpha}^{0}} \\
& -\left\{\left\langle\boldsymbol{\psi}_{2}^{(3)}(j 2 ; j 1 ; r, \boldsymbol{\Omega}),\left[\frac{\partial \boldsymbol{S}(j 3 ; \boldsymbol{\alpha})}{\partial \boldsymbol{\alpha}} \delta \boldsymbol{\alpha}\right] \boldsymbol{\psi}^{(1)}(r, \boldsymbol{\Omega})\right\rangle_{(1)}\right\}_{\boldsymbol{\alpha}^{0}} \\
& -\left\{\left\langle\boldsymbol{\psi}_{3}^{(3)}(j 2 ; j 1 ; r, \boldsymbol{\Omega}),\left[\frac{\partial \boldsymbol{S}(j 3 ; \boldsymbol{\alpha})}{\partial \boldsymbol{\alpha}} \delta \boldsymbol{\alpha}\right] \boldsymbol{\psi}_{1}^{(2)}(j 1 ; r, \boldsymbol{\Omega})\right\rangle_{(1)}\right\}_{\boldsymbol{\alpha}^{0}} \\
& -\left\{\left\langle\boldsymbol{\psi}_{4}^{(3)}(j 2 ; j 1 ; r, \boldsymbol{\Omega}),\left[\frac{\partial \boldsymbol{S}(j 3 ; \boldsymbol{\alpha})}{\partial \boldsymbol{\alpha}} \delta \boldsymbol{\alpha}\right] \boldsymbol{\psi}_{2}^{(2)}(j 1 ; r, \boldsymbol{\Omega})\right\rangle_{(1)}\right\}_{\boldsymbol{\alpha}^{0}},
\end{aligned}
$$

and the "indirect-effect" term is defined as follows:

$$
\begin{aligned}
& \delta\left\{\frac{\partial^{3} L\left(\boldsymbol{\alpha}, \boldsymbol{\varphi} ; \boldsymbol{\psi}^{(1)} ; \boldsymbol{\psi}^{(2)} ; \boldsymbol{\psi}^{(3)}\right)}{\partial t_{j 3} \partial t_{j 2} \partial t_{j 1}}\right\}_{\text {ind }} \\
& \triangleq\left\{\sum_{i=1}^{8}\left\langle\boldsymbol{v}_{i}^{(4)}(j 2 ; j 1 ; r, \boldsymbol{\Omega}), \boldsymbol{r}_{i}^{(4)}(j 3 ; j 2 ; j 1 ; r, \boldsymbol{\Omega})\right\rangle_{(1)}\right\}_{\boldsymbol{\alpha}^{0}} .
\end{aligned}
$$

The quantities appearing on the right side of Equation (29) are defined as follows:

$$
\begin{aligned}
& \boldsymbol{v}^{(4)}(j 2 ; j 1 ; r, \boldsymbol{\Omega}) \triangleq\left[\boldsymbol{v}_{1}^{(4)}(j 2 ; j 1 ; r, \boldsymbol{\Omega}), \cdots, \boldsymbol{v}_{8}^{(4)}(j 2 ; j 1 ; r, \boldsymbol{\Omega})\right]^{\dagger} \\
& \triangleq {\left[\delta \boldsymbol{\varphi}, \delta \boldsymbol{\psi}^{(1)}, \delta \boldsymbol{\psi}_{1}^{(2)}(j 1), \delta \boldsymbol{\psi}_{2}^{(2)}(j 1), \delta \boldsymbol{\psi}_{1}^{(3)}(j 2 ; j 1), \delta \boldsymbol{\psi}_{2}^{(3)}(j 2 ; j 1),\right.} \\
&\left.\delta \boldsymbol{\psi}_{3}^{(3)}(j 2 ; j 1), \delta \boldsymbol{\psi}_{4}^{(3)}(j 2 ; j 1)\right]^{\dagger}, \\
& \boldsymbol{r}^{(4)}(j 3 ; j 2 ; j 1 ; r, \boldsymbol{\Omega}) \triangleq\left[\boldsymbol{r}_{1}^{(4)}(j 3 ; j 2 ; j 1 ; r, \boldsymbol{\Omega}), \cdots, \boldsymbol{r}_{8}^{(4)}(j 3 ; j 2 ; j 1 ; r, \boldsymbol{\Omega})\right]^{\dagger},
\end{aligned}
$$

with components defined as follows:

$$
\boldsymbol{r}_{1}^{(4)}(j 3 ; j 2 ; j 1 ; r, \boldsymbol{\Omega}) \triangleq-\boldsymbol{S}(j 3 ; \alpha) \boldsymbol{\psi}_{1}^{(3)}(j 2 ; j 1 ; r, \boldsymbol{\Omega}),
$$




$$
\begin{gathered}
\boldsymbol{r}_{2}^{(4)}(j 3 ; j 2 ; j 1 ; r, \boldsymbol{\Omega}) \triangleq-\boldsymbol{S}(j 3 ; \boldsymbol{\alpha}) \boldsymbol{\psi}_{2}^{(3)}(j 2 ; j 1 ; r, \boldsymbol{\Omega}), \\
\boldsymbol{r}_{3}^{(4)}(j 3 ; j 2 ; j 1 ; r, \boldsymbol{\Omega}) \triangleq-\boldsymbol{S}(j 3 ; \boldsymbol{\alpha}) \boldsymbol{\psi}_{3}^{(3)}(j 2 ; j 1 ; r, \boldsymbol{\Omega}), \\
\boldsymbol{r}_{4}^{(4)}(j 3 ; j 2 ; j 1 ; r, \boldsymbol{\Omega}) \triangleq-\boldsymbol{S}(j 3 ; \boldsymbol{\alpha}) \boldsymbol{\psi}_{4}^{(3)}(j 2 ; j 1 ; r, \boldsymbol{\Omega}), \\
\boldsymbol{r}_{5}^{(4)}(j 3 ; j 2 ; j 1 ; r, \boldsymbol{\Omega}) \triangleq-\boldsymbol{S}(j 3 ; \boldsymbol{\alpha}) \boldsymbol{\varphi}(r, \boldsymbol{\Omega}), \\
\boldsymbol{r}_{6}^{(4)}(j 3 ; j 2 ; j 1 ; r, \boldsymbol{\Omega}) \triangleq-\boldsymbol{S}(j 3 ; \boldsymbol{\alpha}) \boldsymbol{\psi}^{(1)}(r, \boldsymbol{\Omega}), \\
\boldsymbol{r}_{7}^{(4)}(j 3 ; j 2 ; j 1 ; r, \boldsymbol{\Omega}) \triangleq-\boldsymbol{S}(j 3 ; \boldsymbol{\alpha}) \boldsymbol{\psi}_{1}^{(2)}(j 1 ; r, \boldsymbol{\Omega}), \\
\boldsymbol{r}_{8}^{(4)}(j 3 ; j 2 ; j 1 ; r, \boldsymbol{\Omega}) \triangleq-\boldsymbol{S}(j 3 ; \boldsymbol{\alpha}) \boldsymbol{\psi}_{2}^{(2)}(j 1 ; r, \boldsymbol{\Omega}) .
\end{gathered}
$$

The components of the vector $\boldsymbol{v}^{(4)}(j 2 ; j 1 ; r, \boldsymbol{\Omega})$ defined in Equation (30) are the solutions of the following $4^{\text {th }}$-Level Variational Sensitivity System ( $4^{\text {th }}$-LVSS):

$$
\boldsymbol{F}^{(4)}(j 2 ; j 1 ; r, \boldsymbol{\Omega}) \boldsymbol{v}^{(4)}(j 2 ; j 1 ; r, \boldsymbol{\Omega})=\boldsymbol{q}^{(4)}(j 2 ; j 1 ; r, \boldsymbol{\Omega}),
$$

satisfying the following boundary conditions:

$$
\begin{gathered}
\delta \boldsymbol{\varphi}(r, \boldsymbol{\Omega})=\mathbf{0}, r=r_{d}, \boldsymbol{\Omega} \cdot \mathbf{n}<0, \\
\delta \boldsymbol{\psi}^{(1)}(r, \boldsymbol{\Omega})=\mathbf{0}, r=r_{d}, \boldsymbol{\Omega} \cdot \mathbf{n}>0, \\
\delta \boldsymbol{\psi}_{1}^{(2)}(j 1 ; r, \boldsymbol{\Omega})=\mathbf{0}, r=r_{d}, \boldsymbol{\Omega} \cdot \boldsymbol{n}>0 ; j 1=1, \cdots, J T X, \\
\delta \boldsymbol{\psi}_{2}^{(2)}(j 1 ; r, \boldsymbol{\Omega})=\mathbf{0}, \quad r=r_{d}, \boldsymbol{\Omega} \cdot \mathbf{n}<0 ; j 1=1, \cdots, J T X, \\
\delta \boldsymbol{\psi}_{i}^{(3)}(j 2 ; j 1 ; r, \boldsymbol{\Omega})=\mathbf{0}, r=r_{d} ; \boldsymbol{\Omega} \cdot \boldsymbol{n}<0 ; i=2,3 ; j 1=1, \cdots, J T X ; j 2=1, \cdots, j 1, \\
\delta \boldsymbol{\psi}_{i}^{(3)}(j 2 ; j 1 ; r, \boldsymbol{\Omega})=\mathbf{0}, r=r_{d} ; \boldsymbol{\Omega} \cdot \boldsymbol{n}>0 ; i=1,4 ; j 1=1, \cdots, J T X ; j 2=1, \cdots, j 1 .
\end{gathered}
$$

In Equation (40), the block-matrix $\boldsymbol{F}^{(4)}(j 2 ; j 1 ; r, \boldsymbol{\Omega})$ and the block-vector $\boldsymbol{q}^{(4)}(j 2 ; j 1 ; r, \boldsymbol{\Omega})$ are defined as follows:

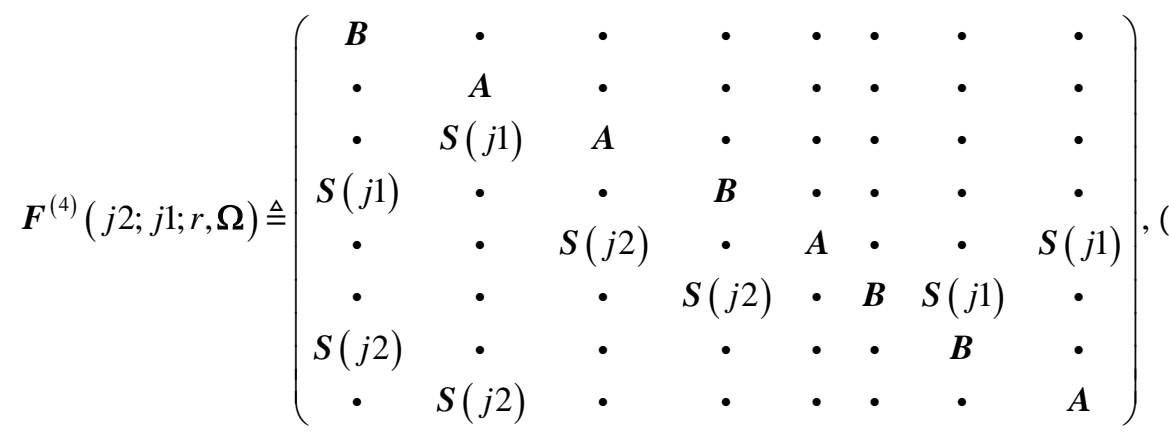

$$
\begin{aligned}
& \boldsymbol{q}^{(4)}(j 2 ; j 1 ; r, \boldsymbol{\Omega}) \\
& \triangleq\left\{\boldsymbol{Q}^{(1)}(\delta \boldsymbol{\alpha}), \boldsymbol{Q}^{(2)}\left(\boldsymbol{\psi}^{(1)} ; \delta \boldsymbol{\alpha}\right), \boldsymbol{Q}_{1}^{(3)}\left[\boldsymbol{\psi}^{(1)} ; \boldsymbol{\psi}_{1}^{(2)}(j 1) ; \delta \boldsymbol{\alpha}\right],\right. \\
& \boldsymbol{Q}_{2}^{(3)}\left[\boldsymbol{\varphi} ; \boldsymbol{\psi}_{2}^{(2)}(j 1) ; \delta \boldsymbol{\alpha}\right], \boldsymbol{Q}_{1}^{(4)}\left[\boldsymbol{\psi}^{(1)} ; \boldsymbol{\psi}^{(2)}(j 1) ; \boldsymbol{\psi}^{(3)}(j 2 ; j 1) ; \delta \boldsymbol{\alpha}\right] \\
& \boldsymbol{Q}_{2}^{(4)}\left[\boldsymbol{\psi}^{(1)} ; \boldsymbol{\psi}^{(2)}(j 1) ; \boldsymbol{\psi}^{(3)}(j 2 ; j 1) ; \delta \boldsymbol{\alpha}\right] \\
& \boldsymbol{Q}_{3}^{(4)}\left[\boldsymbol{\psi}^{(1)} ; \boldsymbol{\psi}^{(2)}(j 1) ; \boldsymbol{\psi}^{(3)}(j 2 ; j 1) ; \delta \boldsymbol{\alpha}\right] \\
&\left.\boldsymbol{Q}_{4}^{(4)}\left[\boldsymbol{\psi}^{(1)} ; \boldsymbol{\psi}^{(2)}(j 1) ; \boldsymbol{\psi}^{(3)}(j 2 ; j 1) ; \delta \boldsymbol{\alpha}\right]\right\}^{\dagger}
\end{aligned}
$$


In order to enhance the visibility of the structure of the $8 \times 8$ matrix $\boldsymbol{F}^{(4)}(j 2 ; j 1 ; r, \boldsymbol{\Omega})$ defined in Equation (46), the zero-valued block-matrix components of this matrix have been represented by dots, while the dependence of the non-zero matrices on the vector of parameters $\boldsymbol{\alpha}$ has been suppressed. In Equation (47), the source-terms $\boldsymbol{Q}^{(1)}(\delta \boldsymbol{\alpha}), \boldsymbol{Q}^{(2)}\left(\psi^{(1)} ; \delta \boldsymbol{\alpha}\right)$, $\boldsymbol{Q}_{i}^{(3)}\left[\boldsymbol{\alpha} ; \boldsymbol{\psi}^{(1)}(r, \boldsymbol{\Omega}) ; \boldsymbol{\psi}_{i}^{(2)}(j 1 ; r, \boldsymbol{\Omega}) ; \delta \boldsymbol{\alpha}\right], i=1,2$ are as follows [2],

$$
\begin{gathered}
\boldsymbol{Q}^{(1)}(\boldsymbol{\alpha}, \boldsymbol{\varphi} ; \delta \boldsymbol{\alpha}) \triangleq-\left\{\frac{\partial\{\boldsymbol{B}(\boldsymbol{\alpha})[\boldsymbol{\varphi}(r, \boldsymbol{\Omega})]\}}{\partial \boldsymbol{\alpha}} \delta \boldsymbol{\alpha}\right\}_{\alpha^{0}}, \\
\boldsymbol{Q}^{(2)}\left(\boldsymbol{\alpha}, \boldsymbol{\psi}^{(1)} ; \delta \boldsymbol{\alpha}\right) \triangleq-\left\{\frac{\partial\left\{\boldsymbol{A}(\boldsymbol{\alpha})\left[\boldsymbol{\psi}^{(1)}(r, \boldsymbol{\Omega})\right]\right\}}{\partial \boldsymbol{\alpha}} \delta \boldsymbol{\alpha}\right\}_{\boldsymbol{\alpha}^{0}}, \\
\boldsymbol{Q}_{1}^{(3)}\left[\boldsymbol{\alpha} ; \boldsymbol{\psi}^{(1)}(r, \boldsymbol{\Omega}) ; \boldsymbol{\psi}_{1}^{(2)}(j 1 ; r, \boldsymbol{\Omega}) ; \delta \boldsymbol{\alpha}\right] \\
\triangleq-\left\{\left[\frac{\partial \boldsymbol{S}(j 1 ; \boldsymbol{\alpha})}{\partial \boldsymbol{\alpha}} \delta \boldsymbol{\alpha}\right] \boldsymbol{\psi}^{(1)}(\boldsymbol{r}, \boldsymbol{\Omega})\right\}_{\boldsymbol{\alpha}^{0}}-\left\{\left[\frac{\partial \boldsymbol{A}(\boldsymbol{\alpha})}{\partial \boldsymbol{\alpha}} \delta \boldsymbol{\alpha}\right] \boldsymbol{\psi}_{1}^{(2)}(j 1 ; r, \boldsymbol{\Omega})\right\}_{\boldsymbol{\alpha}^{0}}, \\
\boldsymbol{Q}_{2}^{(3)}\left[\boldsymbol{\alpha} ; \boldsymbol{\varphi}(r, \boldsymbol{\Omega}) ; \boldsymbol{\psi}_{2}^{(2)}(j 1 ; r, \boldsymbol{\Omega}) ; \delta \boldsymbol{\alpha}\right] \\
\triangleq-\left\{\left[\frac{\partial \boldsymbol{S}(j 1 ; \boldsymbol{\alpha})}{\partial \boldsymbol{\alpha}} \delta \boldsymbol{\alpha}\right] \boldsymbol{\varphi}(\boldsymbol{r}, \boldsymbol{\Omega})\right\}_{\boldsymbol{\alpha}^{0}}-\left\{\left[\frac{\partial \boldsymbol{B}(\boldsymbol{\alpha})}{\partial \boldsymbol{\alpha}} \delta \boldsymbol{\alpha}\right] \boldsymbol{\psi}_{2}^{(2)}(j 1 ; r, \boldsymbol{\Omega})\right\}_{\alpha^{0}}
\end{gathered}
$$

The source-terms $\boldsymbol{Q}_{i}^{(4)}\left[\boldsymbol{\psi}^{(1)} ; \boldsymbol{\psi}^{(2)}(j 1) ; \boldsymbol{\psi}^{(3)}(j 2 ; j 1) ; \delta \boldsymbol{\alpha}\right], i=1,2,3,4$ have the following expressions:

$$
\begin{aligned}
\boldsymbol{Q}_{1}^{(4)} & {\left[\boldsymbol{\psi}^{(1)} ; \boldsymbol{\psi}^{(2)}(j 1) ; \boldsymbol{\psi}^{(3)}(j 2 ; j 1) ; \delta \boldsymbol{\alpha}\right] } \\
\triangleq & -\left[\frac{\partial \boldsymbol{A}(\boldsymbol{\alpha})}{\partial \boldsymbol{\alpha}} \delta \boldsymbol{\alpha}\right] \boldsymbol{\psi}_{1}^{(3)}(j 2 ; j 1)-\left[\frac{\partial \boldsymbol{S}(j 1 ; \boldsymbol{\alpha})}{\partial \boldsymbol{\alpha}} \delta \boldsymbol{\alpha}\right] \boldsymbol{\psi}_{4}^{(3)}(j 2 ; j 1) \\
& -\left[\frac{\partial \boldsymbol{S}(j 2 ; \boldsymbol{\alpha})}{\partial \boldsymbol{\alpha}} \delta \boldsymbol{\alpha}\right] \boldsymbol{\psi}_{1}^{(2)}(j 1 ; r, \boldsymbol{\Omega}), \\
\boldsymbol{Q}_{2}^{(4)} & {\left[\boldsymbol{\psi}^{(1)} ; \boldsymbol{\psi}^{(2)}(j 1) ; \boldsymbol{\psi}^{(3)}(j 2 ; j 1) ; \delta \boldsymbol{\alpha}\right] } \\
\triangleq & -\left[\frac{\partial \boldsymbol{B}(\boldsymbol{\alpha})}{\partial \boldsymbol{\alpha}} \delta \boldsymbol{\alpha}\right] \boldsymbol{\psi}_{2}^{(3)}(j 2 ; j 1)-\left[\frac{\partial \boldsymbol{S}(j 1 ; \boldsymbol{\alpha})}{\partial \boldsymbol{\alpha}} \delta \boldsymbol{\alpha}\right] \boldsymbol{\psi}_{3}^{(3)}(j 2 ; j 1) \\
& -\left[\frac{\partial \boldsymbol{S}(j 2 ; \boldsymbol{\alpha})}{\partial \boldsymbol{\alpha}} \delta \boldsymbol{\alpha}\right] \boldsymbol{\psi}_{2}^{(2)}(j 1 ; r, \boldsymbol{\Omega}), \\
& \boldsymbol{Q}_{3}^{(4)}\left[\boldsymbol{\psi}^{(1)} ; \boldsymbol{\psi}^{(2)}(j 1) ; \boldsymbol{\psi}^{(3)}(j 2 ; j 1) ; \delta \boldsymbol{\alpha}\right] \\
\triangleq & -\left[\frac{\partial \boldsymbol{B}(\boldsymbol{\alpha})}{\partial \boldsymbol{\alpha}} \delta \boldsymbol{\alpha}\right] \boldsymbol{\psi}_{3}^{(3)}(j 2 ; j 1)-\left[\frac{\partial \boldsymbol{S}(j 2 ; \boldsymbol{\alpha})}{\partial \boldsymbol{\alpha}} \delta \boldsymbol{\alpha}\right] \boldsymbol{\varphi}(r, \boldsymbol{\Omega}), \\
\boldsymbol{Q}_{4}^{(4)}\left[\boldsymbol{\psi}^{(1)} ; \boldsymbol{\psi}^{(2)}(j 1) ; \boldsymbol{\psi}^{(3)}(j 2 ; j 1) ; \delta \boldsymbol{\alpha}\right] & \\
\triangleq & -\left[\frac{\partial \boldsymbol{A}(\boldsymbol{\alpha})}{\partial \boldsymbol{\alpha}} \delta \boldsymbol{\alpha}\right] \boldsymbol{\psi}_{4}^{(3)}(j 2 ; j 1)-\left[\frac{\partial \boldsymbol{S}(j 2 ; \boldsymbol{\alpha})}{\partial \boldsymbol{\alpha}} \delta \boldsymbol{\alpha}\right] \boldsymbol{\psi}^{(1)}(r, \boldsymbol{\Omega}) .
\end{aligned}
$$


When only the total microscopic cross sections are considered, the source-terms $\boldsymbol{Q}^{(1)}(\delta \boldsymbol{\alpha}), \quad \boldsymbol{Q}^{(2)}\left(\boldsymbol{\psi}^{(1)} ; \delta \boldsymbol{\alpha}\right), \quad \boldsymbol{Q}_{i}^{(3)}\left[\boldsymbol{\alpha} ; \boldsymbol{\psi}^{(1)}(r, \boldsymbol{\Omega}) ; \boldsymbol{\psi}_{i}^{(2)}(j 1 ; r, \boldsymbol{\Omega}) ; \delta \boldsymbol{\alpha}\right], i=1,2 \quad$ and $\boldsymbol{Q}_{i}^{(4)}\left[\boldsymbol{\psi}^{(1)} ; \boldsymbol{\psi}^{(2)}(j 1) ; \boldsymbol{\psi}^{(3)}(j 2 ; j 1) ; \delta \boldsymbol{\alpha}\right], i=1,2,3,4$, which appear in the definition provided in Equation (47), reduce to the following expressions:

$$
\begin{aligned}
& \boldsymbol{Q}^{(1)}(\delta \boldsymbol{\alpha}) \triangleq-\sum_{j 4=1}^{J T X} \boldsymbol{S}(j 4 ; \boldsymbol{\alpha}) \boldsymbol{\varphi}(r, \boldsymbol{\Omega}) \delta t_{j 4}, \\
& \boldsymbol{Q}^{(2)}\left(\psi^{(1)} ; \delta \boldsymbol{\alpha}\right) \triangleq-\sum_{j 4=1}^{J T X} \boldsymbol{S}(j 4 ; \boldsymbol{\alpha}) \boldsymbol{\psi}^{(1)}(r, \boldsymbol{\Omega}) \delta t_{j 4}, \\
& \boldsymbol{Q}_{1}^{(3)}\left[\boldsymbol{\alpha} ; \boldsymbol{\psi}^{(1)}(r, \boldsymbol{\Omega}) ; \boldsymbol{\psi}_{1}^{(2)}(j 1 ; r, \boldsymbol{\Omega}) ; \delta \boldsymbol{\alpha}\right] \triangleq-\sum_{j 4=1}^{J T X} \boldsymbol{S}(j 4 ; \boldsymbol{\alpha}) \boldsymbol{\psi}_{1}^{(2)}(j 1 ; r, \boldsymbol{\Omega}) \delta t_{j 4} \\
& \boldsymbol{Q}_{2}^{(3)}\left[\boldsymbol{\alpha} ; \boldsymbol{\varphi}(r, \boldsymbol{\Omega}) ; \boldsymbol{\psi}_{2}^{(2)}(j 1 ; r, \boldsymbol{\Omega}) ; \delta \boldsymbol{\alpha}\right] \triangleq-\sum_{j 4=1}^{J T X} \boldsymbol{S}(j 4 ; \boldsymbol{\alpha}) \boldsymbol{\psi}_{2}^{(2)}(j 1 ; r, \boldsymbol{\Omega}) \delta t_{j 4}, \\
& \boldsymbol{Q}_{1}^{(4)}\left[\boldsymbol{\psi}^{(1)} ; \boldsymbol{\psi}^{(2)}(j 1) ; \boldsymbol{\psi}^{(3)}(j 2 ; j 1) ; \delta \boldsymbol{\alpha}\right] \triangleq-\sum_{j 4=1}^{J T X} \boldsymbol{S}(j 4 ; \boldsymbol{\alpha}) \boldsymbol{\psi}_{1}^{(3)}(j 2 ; j 1) \delta t_{j 4}, \\
& \boldsymbol{Q}_{2}^{(4)}\left[\boldsymbol{\psi}^{(1)} ; \boldsymbol{\psi}^{(2)}(j 1) ; \boldsymbol{\psi}^{(3)}(j 2 ; j 1) ; \delta \boldsymbol{\alpha}\right] \triangleq-\sum_{j 4=1}^{J T X} \boldsymbol{S}(j 4 ; \boldsymbol{\alpha}) \boldsymbol{\psi}_{2}^{(3)}(j 2 ; j 1) \delta t_{j 4}, \\
& \boldsymbol{Q}_{3}^{(4)}\left[\boldsymbol{\psi}^{(1)} ; \boldsymbol{\psi}^{(2)}(j 1) ; \boldsymbol{\psi}^{(3)}(j 2 ; j 1) ; \delta \boldsymbol{\alpha}\right] \triangleq-\sum_{j 4=1}^{J T X} \boldsymbol{S}(j 4 ; \boldsymbol{\alpha}) \boldsymbol{\psi}_{3}^{(3)}(j 2 ; j 1) \delta t_{j 4}, \\
& \boldsymbol{Q}_{4}^{(4)}\left[\boldsymbol{\psi}^{(1)} ; \boldsymbol{\psi}^{(2)}(j 1) ; \boldsymbol{\psi}^{(3)}(j 2 ; j 1) ; \delta \boldsymbol{\alpha}\right] \triangleq-\sum_{j 4=1}^{J T X} \boldsymbol{S}(j 4 ; \boldsymbol{\alpha}) \boldsymbol{\psi}_{4}^{(3)}(j 2 ; j 1) \delta t_{j 4} .
\end{aligned}
$$

The huge amounts of large-scale computations that would be needed to solve Equation (40) make it impractical to solve it. To avoid the need for solving Equation (40), the appearance of the vector $\boldsymbol{v}^{(4)}(j 2 ; j 1 ; r, \boldsymbol{\Omega})$ will be eliminated from appearing in the expression on the right-side of Equation (29). This will be accomplished by recasting the inner products that involve $\boldsymbol{v}^{(4)}(j 2 ; j 1 ; r, \boldsymbol{\Omega})$ in terms of equivalent inner products; but these equivalent inner products will involve the solutions of a $4^{\text {th }}$-Level Adjoint Sensitivity System $\left(4^{\text {th }}\right.$-LASS $)$ instead of the components of $\boldsymbol{v}^{(4)}(j 2 ; j 1 ; r, \boldsymbol{\Omega})$. Furthermore, the solutions of the $4^{\text {th }}$-LASS will not depend on the parameter variations. The $4^{\text {th }}$-LASS will be constructed by following a sequence of operations which is similar to the construction of the $3^{\text {rd }}$-LASS [2], and which is described below.

Consider a Hilbert space, denoted as $\mathrm{H}_{(4)}$, comprising elements denoted generically as $\boldsymbol{u}^{(4)}(r, \boldsymbol{\Omega}) \in \mathrm{H}_{(4)}$, which have an "eight-component block-vector" structure of the form $\boldsymbol{u}^{(4)}(r, \boldsymbol{\Omega}) \triangleq\left[\boldsymbol{u}_{1}^{(4)}(r, \boldsymbol{\Omega}), \cdots, \boldsymbol{u}_{8}^{(4)}(r, \boldsymbol{\Omega})\right]^{\dagger}$, with each of the eight vector-components having the following structure: $\boldsymbol{u}_{i}^{(4)}(r, \boldsymbol{\Omega}) \triangleq\left[u_{i}^{(4), 1}(r, \boldsymbol{\Omega}), \cdots, u_{i}^{(4), g}(r, \boldsymbol{\Omega}), \cdots, u_{i}^{(4), G}(r, \boldsymbol{\Omega})\right]^{\dagger}, i=1, \cdots, 8$.

1) Define the inner product in $\mathrm{H}_{(4)}$, denoted as $\left\langle\boldsymbol{u}^{(4)}(r, \boldsymbol{\Omega}), \boldsymbol{w}^{(4)}(r, \boldsymbol{\Omega})\right\rangle_{(4)}$, of two arbitrary elements in $\mathrm{H}_{(4)}$, denoted as $\boldsymbol{u}^{(4)}(r, \boldsymbol{\Omega}) \in \mathrm{H}_{(4)}$ and $\boldsymbol{w}^{(4)}(r, \boldsymbol{\Omega}) \in \mathrm{H}_{(4)}$, and having the block-vector structures $\boldsymbol{w}^{(4)}(r, \boldsymbol{\Omega}) \triangleq\left[\boldsymbol{w}_{1}^{(4)}(r, \boldsymbol{\Omega}), \cdots, \boldsymbol{w}_{8}^{(4)}(r, \boldsymbol{\Omega})\right]^{\dagger}$ and, respectively, 


$$
\begin{aligned}
\boldsymbol{w}_{i}^{(4)}(r, \boldsymbol{\Omega}) \triangleq & {\left[w_{i}^{(4), 1}(r, \boldsymbol{\Omega}), \cdots, w_{i}^{(4), G}(r, \boldsymbol{\Omega})\right]^{\dagger}, i=1, \cdots, 8, \text { as follows: } } \\
& \left\langle\boldsymbol{u}^{(4)}(r, \boldsymbol{\Omega}), \boldsymbol{w}^{(4)}(r, \boldsymbol{\Omega})\right\rangle_{(4)} \triangleq \sum_{i=1}^{8}\left\langle\boldsymbol{u}_{i}^{(4)}(r, \boldsymbol{\Omega}), \boldsymbol{w}_{i}^{(4)}(r, \boldsymbol{\Omega})\right\rangle_{(1)} \\
& =\sum_{i=1}^{8} \sum_{g=1}^{G} \int_{0}^{r_{d}} 4 \pi r^{2} \mathrm{~d} r \int_{4 \pi} \mathrm{d} \boldsymbol{\Omega} u_{i}^{(4), g}(r, \boldsymbol{\Omega}) w_{i}^{(4), g}(r, \boldsymbol{\Omega}) .
\end{aligned}
$$

2) Apply the definition provided in Equation (64) to form the inner product of Equation (40) with a yet undefined vector $\psi^{(4)}(j 3 ; j 2 ; j 1 ; r, \boldsymbol{\Omega}) \triangleq\left[\psi_{1}^{(4)}(j 3 ; j 2 ; j 1 ; r, \boldsymbol{\Omega}), \cdots, \psi_{8}^{(4)}(j 3 ; j 2 ; j 1 ; r, \boldsymbol{\Omega})\right]^{\dagger} \in \mathrm{H}_{(4)}$, to obtain the following relation, evaluated at $\alpha^{0}$ :

$$
\begin{aligned}
& \left\{\left\langle\boldsymbol{\psi}^{(4)}(j 3 ; j 2 ; j 1 ; r, \boldsymbol{\Omega}), \boldsymbol{F}^{(4)}(j 2 ; j 1 ; r, \boldsymbol{\Omega}) \boldsymbol{v}^{(4)}(j 2 ; j 1 ; r, \boldsymbol{\Omega})\right\rangle_{(4)}\right\}_{\alpha^{0}} \\
& =\left\{\left\langle\boldsymbol{\psi}^{(4)}(j 3 ; j 2 ; j 1 ; r, \boldsymbol{\Omega}), \boldsymbol{q}^{(4)}(j 2 ; j 1 ; r, \boldsymbol{\Omega})\right\rangle_{(4)}\right\}_{\boldsymbol{\alpha}^{0}} .
\end{aligned}
$$

3) Use the customary definition of the adjoint operator in $\mathrm{H}_{(4)}$, endowed with the inner product defined in Equation (64) to recast the left side of Equation (65) into the following equivalent form:

$$
\begin{aligned}
& \left\{\left\langle\boldsymbol{\psi}^{(4)}(j 3 ; j 2 ; j 1 ; r, \boldsymbol{\Omega}), \boldsymbol{F}^{(4)}(j 2 ; j 1 ; r, \boldsymbol{\Omega}) \boldsymbol{v}^{(4)}(j 2 ; j 1 ; r, \boldsymbol{\Omega})\right\rangle_{(4)}\right\}_{\alpha^{0}} \\
& =\left\{\left\langle\boldsymbol{v}^{(4)}(j 2 ; j 1 ; r, \boldsymbol{\Omega}),\left[\boldsymbol{F}^{(4)}(j 2 ; j 1 ; r, \boldsymbol{\Omega})\right]^{*} \boldsymbol{\psi}^{(4)}(j 3 ; j 2 ; j 1 ; r, \boldsymbol{\Omega})\right\rangle_{(4)}\right\}_{\boldsymbol{\alpha}^{0}} \\
& +P^{(4)}\left[\delta \boldsymbol{\varphi}, \delta \boldsymbol{\psi}^{(1)} ; \delta \boldsymbol{\psi}^{(2)}(j 1) ; \delta \boldsymbol{\psi}^{(3)}(j 2 ; j 1) ; \boldsymbol{\psi}^{(4)}(j 3 ; j 2 ; j 1)\right],
\end{aligned}
$$

where $P^{(4)}\left[\delta \boldsymbol{\varphi}, \delta \boldsymbol{\psi}^{(1)} ; \delta \boldsymbol{\psi}^{(2)}(j 1) ; \delta \boldsymbol{\psi}^{(3)}(j 2 ; j 1) ; \boldsymbol{\psi}^{(4)}(j 3 ; j 2 ; j 1)\right]$ denotes the corresponding bilinear concomitant evaluated on the PERP sphere's outer boundary $S_{b}$, at $r=r_{d}$, and where the block-matrix operator $\left[\boldsymbol{F}^{(4)}(j 2 ; j 1 ; r, \boldsymbol{\Omega})\right]^{*}$ is the formal adjoint of the block-matrix operator $\boldsymbol{F}^{(4)}(j 2 ; j 1 ; r, \boldsymbol{\Omega})$, having the following structure:

$$
\begin{aligned}
& {\left[\boldsymbol{F}^{(4)}(j 2 ; j 1 ; r, \boldsymbol{\Omega})\right]^{*}}
\end{aligned}
$$

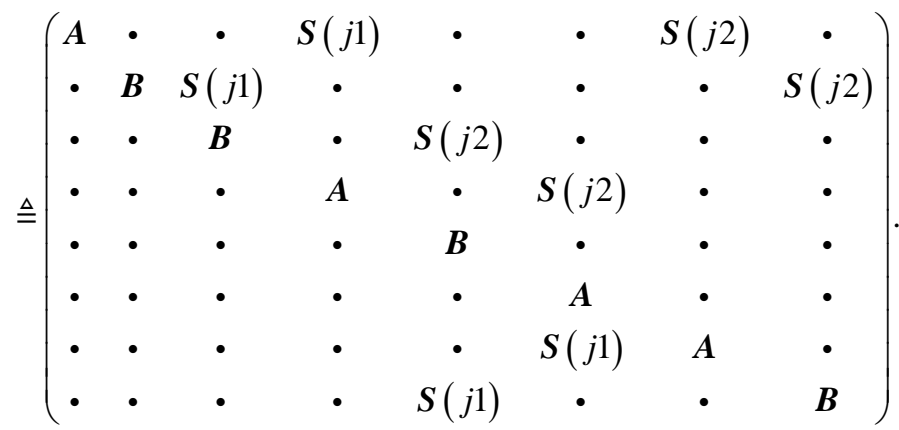

4) Require the first term on the right-side of Equation (66) to represent the same functional as the indirect-effect term defined in Equation (29). This requirement will be satisfied by requiring that the following relations be satisfied by the components of the function $\psi^{(4)}(j 3 ; j 2 ; j 1 ; r, \boldsymbol{\Omega})$ : 


$$
\begin{aligned}
& \left\{\left[\boldsymbol{F}^{(4)}(j 2 ; j 1 ; r, \boldsymbol{\Omega})\right]^{*} \boldsymbol{\psi}^{(4)}(j 3 ; j 2 ; j 1 ; r, \boldsymbol{\Omega})\right\}_{\alpha^{0}}=\boldsymbol{r}^{(4)}(j 3 ; j 2 ; j 1 ; r, \boldsymbol{\Omega}), \\
& j 1=1, \cdots, J T X ; j 2=1, \cdots, j 1 ; j 3=1, \cdots, j 2 .
\end{aligned}
$$

5) Use in Equation (66) the boundary conditions shown in Equations (41), (42), (43), (44) and (45). Furthermore, set to zero the remaining terms in the bilinear concomitant $P^{(4)}\left[\delta \boldsymbol{\varphi}, \delta \boldsymbol{\psi}^{(1)} ; \delta \boldsymbol{\psi}^{(2)}(j 1) ; \delta \boldsymbol{\psi}^{(3)}(j 2 ; j 1) ; \boldsymbol{\psi}^{(4)}(j 3 ; j 2 ; j 1)\right]$ in Equation (66) by requiring the components of the function $\psi^{(4)}(j 3 ; j 2 ; j 1 ; r, \Omega)$ to satisfy the following boundary conditions:

$$
\begin{aligned}
& \boldsymbol{\psi}_{i}^{(4)}(j 3 ; j 2 ; j 1 ; r, \boldsymbol{\Omega})=\mathbf{0}, r=r_{d} ; \boldsymbol{\Omega} \cdot \boldsymbol{n}<0 ; \\
& i=2,3,5,8 ; j 1=1, \cdots, J T X ; j 2=1, \cdots, j 1 ; j 3=1, \cdots, j 2 ; \\
& \boldsymbol{\psi}_{i}^{(4)}(j 3 ; j 2 ; j 1 ; r, \boldsymbol{\Omega})=\mathbf{0}, r=r_{d} ; \boldsymbol{\Omega} \cdot \boldsymbol{n}>0 ; \\
& i=1,4,6,7 ; j 1=1, \cdots, J T X ; j 2=1, \cdots, j 1 ; j 3=1, \cdots, j 2 .
\end{aligned}
$$

6) The boundary conditions shown in Equation (69) complete the well-posed definition of the $4^{\text {th }}$-level adjoint function $\psi^{(4)}(j 3 ; j 2 ; j 1 ; r, \Omega) \in \mathrm{H}_{(4)}$ as the solution of Equations (68) and (69), which are called "the Fourth-Level Adjoint Sensitivity System ( $4^{\text {th }}$-LASS)". The reason for calling this system "Fourth-LeveP" (as opposed to "Fourth-Order") stems from the fact that this system does not involve any $2^{\text {nd }}$ - $3^{\text {rd }}$ - and/or $4^{\text {th }}$-order differentials or derivatives of the dependent variables (i.e., state functions) even though the solution of the $4^{\text {th }}$-LASS is used for computing, efficiently and exactly, the $4^{\text {th }}$-order sensitivities of the response with respect to the model parameters.

7) Use Equations (66) through (69) in Equation (29) to obtain the following alternative expression for the indirect-effect term $\delta\left\{\partial^{3} L\left(\boldsymbol{\alpha}, \boldsymbol{\varphi} ; \boldsymbol{\psi}^{(1)} ; \boldsymbol{\psi}^{(2)} ; \boldsymbol{\psi}^{(3)}\right) / \partial t_{j 3} \partial t_{j 2} \partial t_{j 1}\right\}_{\text {ind }}$ in terms of the $4^{\text {th }}$-level adjoint function $\psi^{(4)}(j 3 ; j 2 ; j 1 ; r, \boldsymbol{\Omega}) \in \mathrm{H}_{(4)}$ :

$$
\begin{aligned}
& \delta\left\{\frac{\partial^{3} L\left(\boldsymbol{\alpha}, \boldsymbol{\varphi} ; \boldsymbol{\psi}^{(1)} ; \boldsymbol{\psi}^{(2)} ; \boldsymbol{\psi}^{(3)}\right)}{\partial t_{j 3} \partial t_{j 2} \partial t_{j 1}}\right\}_{\text {ind }} \\
& =\sum_{i=1}^{8}\left\{\left\langle\boldsymbol{\psi}_{i}^{(4)}(j 3 ; j 2 ; j 1 ; r, \boldsymbol{\Omega}), \boldsymbol{q}_{i}^{(4)}(j 2 ; j 1 ; r, \boldsymbol{\Omega})\right\rangle_{(1)}\right\}_{\boldsymbol{\alpha}^{0}} \\
& =\sum_{j 4=1}^{T P}\left\{\frac{\partial^{4} L\left(\boldsymbol{\alpha}, \boldsymbol{\varphi} ; \boldsymbol{\psi}^{(1)} ; \boldsymbol{\psi}^{(2)} ; \boldsymbol{\psi}^{(3)}\right)}{\partial \alpha_{j 4} \partial t_{j 3} \partial t_{j 2} \partial t_{j 1}} \delta \alpha_{j 4}\right\}_{\alpha^{0}}, \\
& j 1=1, \cdots, J T X ; j 2=1, \cdots, j 1 ; j 3=1, \cdots, j 2 .
\end{aligned}
$$

8) The differential expression for the indirect-effect term $\delta\left\{\partial^{3} L\left(\alpha, \boldsymbol{\varphi} ; \boldsymbol{\psi}^{(1)} ; \boldsymbol{\psi}^{(2)} ; \boldsymbol{\psi}^{(3)}\right) / \partial t_{j 3} \partial t_{j 2} \partial t_{j 1}\right\}_{\text {ind }} \quad$ provided in Equation (70) comprises all of the $4^{\text {th }}$-order partial sensitivities that involve the group-averaged microscopic total cross sections, i.e., $4^{\text {th }}$-order partial response sensitivities with respect to the total microscopic cross sections (indexed by $j 1, j 2$ and $j 3$ ) and with respect to the other model parameters (indexed by $j 4$ ). 
9) In particular, if only the variations in the total microscopic cross sections are considered, then the direct-effect term defined in Equation (28) vanishes and the indirect-effect term in Equation (70) reduces to the following particular form:

$$
\begin{aligned}
& \delta\left\{\frac{\partial^{3} L\left(\boldsymbol{\alpha}, \boldsymbol{\varphi} ; \boldsymbol{\psi}^{(1)} ; \boldsymbol{\psi}^{(2)} ; \boldsymbol{\psi}^{(3)}\right)}{\partial t_{j 3} \partial t_{j 2} \partial t_{j 1}}\right\}_{\text {ind }} \\
& =\sum_{i=1}^{8}\left\{\left\langle\boldsymbol{\psi}_{i}^{(4)}(j 3 ; j 2 ; j 1 ; r, \boldsymbol{\Omega}), \boldsymbol{q}_{i}^{(4)}(j 2 ; j 1 ; r, \boldsymbol{\Omega})\right\rangle_{(1)}\right\}_{\boldsymbol{\alpha}^{0}} \\
& =\sum_{j 4=1}^{J T X}\left\{\frac{\partial^{4} L\left(\alpha, \boldsymbol{\varphi} ; \boldsymbol{\psi}^{(1)} ; \boldsymbol{\psi}^{(2)} ; \boldsymbol{\psi}^{(3)}\right)}{\partial t_{j 4} \partial t_{j 3} \partial t_{j 2} \partial t_{j 1}} \delta t_{j 4}\right\}_{\alpha^{0}}, \\
& j 1=1, \cdots, J T X ; j 2=1, \cdots, j 1 ; j 3=1, \cdots, j 2 .
\end{aligned}
$$

10) Identifying the specific quantity that multiplies the specific variation $\delta t_{j 4}$ provides the following expression for the $4^{\text {th }}$-order response sensitivities which involve only the group-averaged total microscopic cross sections:

$$
\begin{aligned}
& \left\{\frac{\partial^{4} L\left(\boldsymbol{\alpha}, \boldsymbol{\varphi} ; \boldsymbol{\psi}^{(1)} ; \boldsymbol{\psi}^{(2)} ; \boldsymbol{\psi}^{(3)} ; \boldsymbol{\psi}^{(4)}\right)}{\partial t_{j 4} \partial t_{j 3} \partial t_{j 2} \partial t_{j 1}}\right\}_{\boldsymbol{\alpha}^{0}} \\
& =-\left\{\left\langle\boldsymbol{\psi}_{1}^{(4)}(j 3 ; j 2 ; j 1 ; r, \boldsymbol{\Omega}), \boldsymbol{S}(j 4 ; \boldsymbol{\alpha}) \boldsymbol{\varphi}(r, \boldsymbol{\Omega})\right\rangle_{(1)}\right\}_{\alpha^{0}} \\
& -\left\{\left\langle\boldsymbol{\psi}_{2}^{(4)}(j 3 ; j 2 ; j 1 ; r, \boldsymbol{\Omega}), \boldsymbol{S}(j 4 ; \boldsymbol{\alpha}) \boldsymbol{\psi}^{(1)}(r, \boldsymbol{\Omega})\right\rangle_{(1)}\right\}_{\alpha^{0}} \\
& -\left\{\left\langle\boldsymbol{\psi}_{3}^{(4)}(j 3 ; j 2 ; j 1 ; r, \boldsymbol{\Omega}), \boldsymbol{S}(j 4 ; \alpha) \psi_{1}^{(2)}(j 1 ; r, \boldsymbol{\Omega})\right\rangle_{(1)}\right\}_{\alpha^{0}} \\
& -\left\{\left\langle\psi_{4}^{(4)}(j 3 ; j 2 ; j 1 ; r, \Omega), \boldsymbol{S}(j 4 ; \boldsymbol{\alpha}) \psi_{2}^{(2)}(j 1 ; r, \Omega)\right\rangle_{(1)}\right\}_{\boldsymbol{\alpha}^{0}} \\
& -\left\{\left\langle\boldsymbol{\psi}_{5}^{(4)}(j 3 ; j 2 ; j 1 ; r, \boldsymbol{\Omega}), \boldsymbol{S}(j 4 ; \boldsymbol{\alpha}) \boldsymbol{\psi}_{1}^{(3)}(j 2 ; j 1 ; r, \boldsymbol{\Omega})\right\rangle_{(1)}\right\}_{\alpha^{0}} \\
& -\left\{\left\langle\boldsymbol{\psi}_{6}^{(4)}(j 3 ; j 2 ; j 1 ; r, \boldsymbol{\Omega}), \boldsymbol{S}(j 4 ; \boldsymbol{\alpha}) \boldsymbol{\psi}_{2}^{(3)}(j 2 ; j 1 ; r, \boldsymbol{\Omega})\right\rangle_{(1)}\right\}_{\boldsymbol{\alpha}^{0}} \\
& -\left\{\left\langle\boldsymbol{\psi}_{7}^{(4)}(j 3 ; j 2 ; j 1 ; r, \boldsymbol{\Omega}), \boldsymbol{S}(j 4 ; \boldsymbol{\alpha}) \boldsymbol{\psi}_{3}^{(3)}(j 2 ; j 1 ; r, \boldsymbol{\Omega})\right\rangle_{(1)}\right\}_{\alpha^{0}} \\
& -\left\{\left\langle\boldsymbol{\psi}_{8}^{(4)}(j 3 ; j 2 ; j 1 ; r, \boldsymbol{\Omega}), \boldsymbol{S}(j 4 ; \boldsymbol{\alpha}) \boldsymbol{\psi}_{4}^{(3)}(j 2 ; j 1 ; r, \boldsymbol{\Omega})\right\rangle_{(1)}\right\}_{\alpha^{0}}, \\
& \text { for } j 1=1, \cdots, J T X ; j 2=1, \cdots, j 1 ; j 3=1, \cdots, j 2 ; j 4=1, \cdots, j 3 \text {, }
\end{aligned}
$$

where the $4^{\text {th }}$-level adjoint function $\psi^{(4)}(j 3 ; j 2 ; j 1 ; r, \Omega)$ is the solution of the $4^{\text {th }}$-LASS defined by Equations (68) and (69). In component form, the $4^{\text {th }}$-LASS is solved in the following sequence:

$$
\begin{gathered}
\left\{\boldsymbol{B}(\boldsymbol{\alpha}) \boldsymbol{\psi}_{5}^{(4)}(j 3 ; j 2 ; j 1 ; r, \boldsymbol{\Omega})\right\}_{\alpha^{0}}=-\{\boldsymbol{S}(j 3 ; \boldsymbol{\alpha}) \boldsymbol{\varphi}(r, \boldsymbol{\Omega})\}_{\alpha^{0}}, \\
\boldsymbol{\psi}_{5}^{(4)}(j 3 ; j 2 ; j 1 ; r, \boldsymbol{\Omega})=\mathbf{0}, r=r_{d} ; \boldsymbol{\Omega} \cdot \boldsymbol{n}<0 ; \\
j 1=1, \cdots, J T X ; j 2=1, \cdots, j 1 ; j 3=1, \cdots, j 2 ;
\end{gathered}
$$




$$
\begin{aligned}
& \left\{\boldsymbol{A}(\boldsymbol{\alpha}) \boldsymbol{\psi}_{6}^{(4)}(j 3 ; j 2 ; j 1 ; r, \boldsymbol{\Omega})\right\}_{\alpha^{0}}=-\left\{\boldsymbol{S}(j 3 ; \boldsymbol{\alpha}) \psi^{(1)}(r, \boldsymbol{\Omega})\right\}_{\alpha^{0}}, \\
& \boldsymbol{\psi}_{6}^{(4)}(j 3 ; j 2 ; j 1 ; r, \boldsymbol{\Omega})=\mathbf{0}, r=r_{d} ; \boldsymbol{\Omega} \cdot \boldsymbol{n}>0 ; \\
& j 1=1, \cdots, J T X ; j 2=1, \cdots, j 1 ; j 3=1, \cdots, j 2 . \\
& \left\{\boldsymbol{A}(\boldsymbol{\alpha}) \boldsymbol{\psi}_{7}^{(4)}(j 3 ; j 2 ; j 1 ; r, \boldsymbol{\Omega})\right\}_{\boldsymbol{\alpha}^{0}} \\
& =-\left\{\boldsymbol{S}(j 1 ; \boldsymbol{\alpha}) \boldsymbol{\psi}_{6}^{(4)}(j 3 ; j 2 ; j 1 ; r, \boldsymbol{\Omega})\right\}_{\boldsymbol{\alpha}^{0}}-\left\{\boldsymbol{S}(j 3 ; \boldsymbol{\alpha}) \boldsymbol{\psi}_{1}^{(2)}(j 1 ; r, \boldsymbol{\Omega})\right\}_{\alpha^{0}}, \\
& \boldsymbol{\psi}_{7}^{(4)}(j 3 ; j 2 ; j 1 ; r, \boldsymbol{\Omega})=\mathbf{0}, r=r_{d} ; \boldsymbol{\Omega} \cdot \boldsymbol{n}>0 ; \\
& j 1=1, \cdots, J T X ; j 2=1, \cdots, j 1 ; j 3=1, \cdots, j 2 . \\
& \left\{\boldsymbol{B}(\boldsymbol{\alpha}) \boldsymbol{\psi}_{8}^{(4)}(j 3 ; j 2 ; j 1 ; r, \boldsymbol{\Omega})\right\}_{\alpha^{0}} \\
& =-\left\{\boldsymbol{S}(j 1 ; \alpha) \psi_{5}^{(4)}(j 3 ; j 2 ; j 1 ; r, \boldsymbol{\Omega})\right\}_{\alpha^{0}}-\left\{\boldsymbol{S}(j 3 ; \boldsymbol{\alpha}) \psi_{2}^{(2)}(j 1 ; r, \boldsymbol{\Omega})\right\}_{\alpha^{0}} \text {. } \\
& \boldsymbol{\psi}_{8}^{(4)}(j 3 ; j 2 ; j 1 ; r, \boldsymbol{\Omega})=\mathbf{0}, r=r_{d} ; \boldsymbol{\Omega} \cdot \boldsymbol{n}<0 ; \\
& j 1=1, \cdots, J T X ; j 2=1, \cdots, j 1 ; j 3=1, \cdots, j 2 \text {; } \\
& \left\{\boldsymbol{B}(\boldsymbol{\alpha}) \boldsymbol{\psi}_{3}^{(4)}(j 3 ; j 2 ; j 1 ; r, \Omega)\right\}_{\boldsymbol{\alpha}^{0}} \\
& =-\left\{\boldsymbol{S}(j 2 ; \boldsymbol{\alpha}) \boldsymbol{\psi}_{5}^{(4)}(j 3 ; j 2 ; j 1 ; r, \boldsymbol{\Omega})\right\}_{\alpha^{0}}-\left\{\boldsymbol{S}(j 3 ; \boldsymbol{\alpha}) \boldsymbol{\psi}_{3}^{(3)}(j 2 ; j 1 ; r, \boldsymbol{\Omega})\right\}_{\alpha^{0}} \text {, } \\
& \boldsymbol{\psi}_{3}^{(4)}(j 3 ; j 2 ; j 1 ; r, \boldsymbol{\Omega})=\mathbf{0}, r=r_{d} ; \boldsymbol{\Omega} \cdot \boldsymbol{n}<0 ; \\
& j 1=1, \cdots, J T X ; j 2=1, \cdots, j 1 ; j 3=1, \cdots, j 2 \text {; } \\
& \left\{\boldsymbol{A}(\boldsymbol{\alpha}) \boldsymbol{\psi}_{4}^{(4)}(j 3 ; j 2 ; j 1 ; r, \boldsymbol{\Omega})\right\}_{\alpha^{0}} \\
& =-\left\{\boldsymbol{S}(j 2 ; \boldsymbol{\alpha}) \boldsymbol{\psi}_{6}^{(4)}(j 3 ; j 2 ; j 1 ; r, \boldsymbol{\Omega})\right\}_{\alpha^{0}}-\left\{\boldsymbol{S}(j 3 ; \alpha) \boldsymbol{\psi}_{4}^{(3)}(j 2 ; j 1 ; r, \boldsymbol{\Omega})\right\}_{\alpha^{0}} \text {, } \\
& \boldsymbol{\psi}_{4}^{(4)}(j 3 ; j 2 ; j 1 ; r, \boldsymbol{\Omega})=\mathbf{0}, r=r_{d} ; \boldsymbol{\Omega} \cdot \boldsymbol{n}>0 ; \\
& j 1=1, \cdots, J T X ; j 2=1, \cdots, j 1 ; j 3=1, \cdots, j 2 . \\
& \left\{\boldsymbol{A}(\boldsymbol{\alpha}) \boldsymbol{\psi}_{1}^{(4)}(j 3 ; j 2 ; j 1 ; r, \boldsymbol{\Omega})\right\}_{\alpha^{0}}=-\left\{\boldsymbol{S}(j 1 ; \boldsymbol{\alpha}) \boldsymbol{\psi}_{4}^{(4)}(j 3 ; j 2 ; j 1 ; r, \boldsymbol{\Omega})\right\}_{\boldsymbol{\alpha}^{0}} \\
& -\left\{\boldsymbol{S}(j 2 ; \boldsymbol{\alpha}) \boldsymbol{\psi}_{7}^{(4)}(j 3 ; j 2 ; j 1 ; r, \boldsymbol{\Omega})\right\}_{\alpha^{0}}-\left\{\boldsymbol{S}(j 3 ; \boldsymbol{\alpha}) \boldsymbol{\psi}_{1}^{(3)}(j 2 ; j 1 ; r, \boldsymbol{\Omega})\right\}_{\boldsymbol{\alpha}^{0}}, \\
& \boldsymbol{\psi}_{1}^{(4)}(j 3 ; j 2 ; j 1 ; r, \boldsymbol{\Omega})=\mathbf{0}, r=r_{d} ; \boldsymbol{\Omega} \cdot \boldsymbol{n}>0 ; \\
& j 1=1, \cdots, J T X ; j 2=1, \cdots, j 1 ; j 3=1, \cdots, j 2 \text {. } \\
& \left\{\boldsymbol{B}(\boldsymbol{\alpha}) \boldsymbol{\psi}_{2}^{(4)}(j 3 ; j 2 ; j 1 ; r, \boldsymbol{\Omega})\right\}_{\boldsymbol{\alpha}^{0}}=-\left\{\boldsymbol{S}(j 1 ; \boldsymbol{\alpha}) \boldsymbol{\psi}_{3}^{(4)}(j 3 ; j 2 ; j 1 ; r, \boldsymbol{\Omega})\right\}_{\alpha^{0}} \\
& -\left\{\boldsymbol{S}(j 2 ; \boldsymbol{\alpha}) \boldsymbol{\psi}_{8}^{(4)}(j 3 ; j 2 ; j 1 ; r, \boldsymbol{\Omega})\right\}_{\alpha^{0}}-\left\{\boldsymbol{S}(j 3 ; \boldsymbol{\alpha}) \boldsymbol{\psi}_{2}^{(3)}(j 2 ; j 1 ; r, \boldsymbol{\Omega})\right\}_{\boldsymbol{\alpha}^{0}} \text {, } \\
& \boldsymbol{\psi}_{2}^{(4)}(j 3 ; j 2 ; j 1 ; r, \boldsymbol{\Omega})=\mathbf{0}, r=r_{d} ; \boldsymbol{\Omega} \cdot \boldsymbol{n}<0 ; \\
& j 1=1, \cdots, J T X ; j 2=1, \cdots, j 1 ; j 3=1, \cdots, j 2 .
\end{aligned}
$$

Thus, as indicated by Equations (73)-(88), computing the components of the $4^{\text {th }}$-level adjoint function $\psi^{(4)}(j 3 ; j 2 ; j 1 ; r, \boldsymbol{\Omega})$ requires four forward-like PARTISN computations and four adjoint-like PARTISN computations.

\subsubsection{Comparison of Computational Requirements}

The numbers of sensitivities of the PERP leakage response with respect to the 
total microscopic cross sections are as follows: 1) 180 first-order sensitivities; 2) 32,400 second-order sensitivities, of which 16,290 are distinct; and 3) 5,832,000 third-order sensitivities, of which 988,260 are distinct; 4) 1,049,760,000 fourthorder sensitivities, of which 45,212,895 are distinct.

Using a DELL computer (AMD FX-8350) with an 8-core processor, the CPUtime for a typical adjoint computation using PARTISN with an angular quadrature of $\mathrm{S}_{32}(\mathrm{ISN}=32)$ is ca. 20 seconds, while the CPU-time computing the integrals over the various adjoint functions which appear in the definition of the respective sensitivity in Equation (72) is ca. 0.003 seconds.

The comparison of the CPU-times for all of the distinct $4^{\text {th }}$-order sensitivities required by applying the $4^{\text {th }}$-CASAM, versus using the FSAM or the FD-approximations is presented in Table 1. Based on Equations (11) and (12), the number of forward computations needed to obtain all of the 45,212,895 distinct 4 th-order sensitivities using the FD-approximation method is $723,404,160$ [= $180 \times 4$ forward PARTISN computations for the unmixed $4^{\text {th }}$-order sensitivities $+(45,212,895-180) \times 16$ forward PARTISN computations for the mixed 4 th-order sensitivities].

Based on Equations (14) and (15), the total number of forward computations needed to obtain all of the distinct $4^{\text {th }}$-order sensitivities $\partial^{4} L(\alpha) / \partial t_{j 4} \partial t_{j 3} \partial t_{j 2} \partial t_{j 1}$ using the FSAM method is $46,217,625$, which is the result of $180+180(180+1) / 2+180(180+1)(180+2) / 3 !+180(180+1)(180+2)(180+3) / 4 !$.

Based on Equations (73)-(88), a total number of 2,075,341 adjoint computations is needed to obtain all of the distinct 4 th-order sensitivities $\partial^{4} L(\alpha) / \partial t_{j 4} \partial t_{j 3} \partial t_{j 2} \partial t_{j 1}$ by the 4 th-CASAM method, comprising the following large-scale computations:

1) 33,301 adjoint PARTISN computations to obtain $\psi^{(1)}(r, \Omega), \psi_{1}^{(2)}(j 1 ; r, \Omega)$; $\boldsymbol{\psi}_{2}^{(2)}(j 1 ; r, \boldsymbol{\Omega}), \quad \boldsymbol{\psi}_{1}^{(3)}(j 2 ; j 1 ; r, \boldsymbol{\Omega}), \quad \boldsymbol{\psi}_{2}^{(3)}(j 2 ; j 1 ; r, \boldsymbol{\Omega}), \boldsymbol{\psi}_{3}^{(3)}(j 2 ; j 1 ; r, \boldsymbol{\Omega})$ and $\boldsymbol{\psi}_{4}^{(3)}(j 2 ; j 1 ; r, \boldsymbol{\Omega})$, as described in [2];

2) 988,260 adjoint-like PARTISN computations to obtain $\psi_{1}^{(4)}(j 3 ; j 2 ; j 1 ; r, \Omega)$;

3) 988,260 forward-like PARTISN computations to obtain $\psi_{2}^{(4)}(j 3 ; j 2 ; j 1 ; r, \Omega)$;

4) 16,290 forward-like PARTISN computations to obtain $\psi_{3}^{(4)}(j 3 ; j 2 ; j 1 ; r, \Omega)$;

5) 16,290 adjoint-like PARTISN computations to obtain $\psi_{4}^{(4)}(j 3 ; j 2 ; j 1 ; r, \Omega)$;

6) 180 forward-like PARTISN computations to obtain $\psi_{5}^{(4)}(j 3 ; j 2 ; j 1 ; r, \Omega)$;

7) 180 adjoint-like PARTISN computations to obtain $\psi_{6}^{(4)}(j 3 ; j 2 ; j 1 ; r, \Omega)$;

8) 16,290 adjoint-like PARTISN computations to obtain $\psi_{7}^{(4)}(j 3 ; j 2 ; j 1 ; r, \Omega)$;

9) 16,290 forward-like PARTISN computations to obtain $\psi_{8}^{(4)}(j 3 ; j 2 ; j 1 ; r, \Omega)$.

Table 1. CPU-times for computing $\partial^{4} L\left(\boldsymbol{\alpha}, \boldsymbol{\varphi} ; \boldsymbol{\psi}^{(1)} ; \boldsymbol{\psi}^{(2)} ; \boldsymbol{\psi}^{(3)} ; \boldsymbol{\psi}^{(4)}\right) / \partial t_{j 4} \partial t_{j 3} \partial t_{j 2} \partial t_{j 1}$ for $j 1=1, \cdots, J T X ; j 2=1, \cdots, j 1 ; j 3=1, \cdots, j 2 ; j 4=1, \cdots, j 3$.

\begin{tabular}{|c|c|c|}
\hline FD-approximation & FSAM & 4th-CASAM \\
\hline Nr. Forward comp. $=723,404,160$ & Nr. Forward comp. $=46,217,625$ & Nr. Adjoint comp. $=2,075,341$ \\
\hline $\mathrm{ISN}=32$ & $\mathrm{ISN}=32$ & $\mathrm{ISN}=32$ \\
\hline CPU time $\approx 9,042,442$ Hours & $\mathrm{CPU}$ time $=577,720$ Hours & CPU time $\approx 11,568$ Hours \\
\hline
\end{tabular}


As shown in Table 1 , the $4^{\text {th }}$-CASAM method (which is by far the most efficient method) needs ca. 11,568 hours CPU-time for obtaining all of the distinct $4^{\text {th }}$-order sensitivities $\partial^{4} L(\alpha) / \partial t_{j 4} \partial t_{j 3} \partial t_{j 2} \partial t_{j 1}$. The computations of these sensitivities must therefore be prioritized, in the order of their magnitudes. Based on the magnitudes of the $1^{\text {st }}-, 2^{\text {nd }}$ - and $3^{\text {rd }}$-order sensitivities, which were computed in previous works [2] [10], the most important $4^{\text {th }}$-order sensitivities are expected to include the 180 unmixed 4 th-order sensitivities, namely,

$\partial^{4} L(\alpha) /\left(\partial t_{j}\right)^{4}, j=1, \cdots, J T X$, and the mixed 4 th-order sensitivity that corresponds to the largest $3^{\text {rd }}$-order one, namely, for

$S^{(3)}\left(\sigma_{t, i=1}^{g=30}, \sigma_{t, i=6}^{g=30}, \sigma_{t, i=6}^{g=30}\right)=-1.88 \times 10^{5}$ [10]. The total number of adjoint computations needed for computing the 180 unmixed fourth-order sensitivities is 2521, comprising the following large-scale computations:

1) 1 adjoint computations for computing $\psi^{(1)}(r, \Omega)$;

2) $180 \times 2$ adjoint computations for computing

$\psi_{i}^{(2)}(j ; r, \Omega), j=1, \cdots, 180 ; i=1,2$;

3) $180 \times 4$ adjoint computations for computing $\psi_{i}^{(3)}(j ; j ; r, \Omega)$, where $j=1, \cdots, 180 ; i=1,2,3,4$;

4) $180 \times 8$ adjoint computations for computing $\psi_{i}^{(4)}(j ; j ; j ; r, \Omega)$, where $j=1, \cdots, 180 ; i=1, \cdots, 8$.

Similarly, a total number of 14 adjoint computations are needed for computing the mixed $4^{\text {th }}$-order sensitivity that corresponds to the largest $3^{\text {rd }}$-order one. The total CPU-time is approximately 14 Hours for computing: 1) the 2535 (= $2521+14$ ) adjoint functions using PARTISN with an angular quadrature of $\mathrm{S}_{32}$; and 2) the integrals over the various adjoint functions to obtain the 181 (i.e., 180 unmixed and 1 mixed) fourth-order sensitivities.

\section{Concluding Remarks}

This work has presented the derivation of the exact mathematical expressions of the fourth-order sensitivities of the PERP leakage response with respect to the total microscopic cross sections. The formulas derived in this work are valid not only for the PERP benchmark but can also be used for computing the $4^{\text {th }}$-order sensitivities of the leakage response of any nuclear system involving fissionable material and internal or external neutron sources. Subsequent work [4] will use the mathematical expressions obtained in this work to compute exactly and efficiently the numerical values of the unmixed and the largest mixed fourth-order sensitivities of the PERP leakage response with respect to the total microscopic cross sections, and use them for a fourth-order uncertainty analysis of the PERP benchmark's leakage response.

\section{Conflicts of Interest}

The authors declare no conflicts of interest regarding the publication of this paper.

\section{References}

[1] Cacuci D.G. (2021) Fourth-Order Comprehensive Adjoint Sensitivity Analysis ( $4^{\text {th }}$ - 
CASAM) of Response-Coupled Linear Forward/Adjoint Systems: I. Theoretical Framework, Energies; Accepted.

[2] Cacuci, D.G. and Fang, R. (2021) Fourth-Order Adjoint Sensitivity Analysis of an OECD/NEA Reactor Physics Benchmark: I. Mathematical Expressions and CPUTime Comparisons for Computing $1^{\text {st }}-2^{\text {nd }}$ and $3^{\text {rd }}$-Order Sensitivities. American Journal of Computational Mathematics, 2, 94-132.

https://doi.org/10.4236/ajcm.2021.112009

[3] Valentine, T.E. (2006) Polyethylene-Reflected Plutonium Metal Sphere Subcritical Noise Measurements, SUB-PU-METMIXED-001. International Handbook of Evaluated Criticality Safety Benchmark Experiments, NEA/NSC/DOC(95)03/I-IX, Organization for Economic Co-Operation and Development. Nuclear Energy Agency, Paris.

[4] Fang, R. and Cacuci, D.G. (2021) Fourth-Order Adjoint Sensitivity and Uncertainty Analysis of an OECD/NEA Reactor Physics Benchmark. Annals of Nuclear Energy, to be Submitted.

[5] Cacuci, D.G., Fang, R. and Favorite, J.A. (2019) Comprehensive Second-Order Adjoint Sensitivity Analysis Methodology (2nd-ASAM) Applied to a Subcritical Experimental Reactor Physics Benchmark: I. Effects of Imprecisely Known Microscopic Total and Capture Cross Sections. Energies, 12, 4219.

https://doi.org/10.3390/en12214219

[6] Fang, R. and Cacuci, D.G. (2020) Third-Order Adjoint Sensitivity Analysis of an OECD/NEA Reactor Physics Benchmark: III. Response Moments. American Journal of Computational Mathematics (AJCM), 10, 559-570.

https://www.scirp.org/journal/ajcm https://doi.org/10.4236/ajcm.2020.104031

[7] Alcouffe, R.E., Baker, R.S., Dahl, J.A., Turner, S.A. and Ward, R. (2008) PARTISN: A Time-Dependent, Parallel Neutral Particle Transport Code System. LA-UR-0807258, Los Alamos National Laboratory, Los Alamos, NM.

[8] Wilson, W.B., Perry, R.T., Shores, E.F., Charlton, W.S., Parish, T.A., Estes, G.P., Brown, T.H., Arthur, E.D., Bozoian, M., England, T.R., et al. (2002) SOURCES4C: A Code for Calculating $(\alpha, n)$, Spontaneous Fission, and Delayed Neutron Sources and Spectra. Proceedings of the American Nuclear Society/Radiation Protection and Shielding Division 12th Biennial Topical Meeting, Santa Fe, NM, 14-18 April 2002.

[9] Conlin, J.L, Parsons, D.K., Gardiner, S.J., Beth Lee, M.G.M. and White, M.C. (2013) MENDF71X: Multigroup Neutron Cross-Section Data Tables Based upon ENDF/BVII.1X. Los Alamos National Laboratory Report LA-UR-15-29571; Los Alamos National Laboratory: Los Alamos, NM, USA.

[10] Fang, R. and Cacuci, D.G. (2020) Third-Order Adjoint Sensitivity Analysis of an OECD/NEA Reactor Physics Benchmark: II. Computed Sensitivities. American Journal of Computational Mathematics (AJCM), 10, 529-558.

https://www.scirp.org/journal/ajcm

https://doi.org/10.4236/ajcm.2020.104030 


\section{Appendix}

The spherical polyethylene-reflected plutonium (acronym: PERP) benchmark comprises a metallic inner sphere ("core") containing the following 4 isotopes: Isotope $1\left({ }^{239} \mathrm{Pu}\right)$, Isotope $2\left({ }^{240} \mathrm{Pu}\right)$, Isotope $3\left({ }^{69} \mathrm{Ga}\right)$ and Isotope $4\left({ }^{71} \mathrm{Ga}\right)$. This core (which is designated as "material 1") is surrounded by a spherical shell of polyethylene (designated as "material 2"), containing two isotopes, designated as Isotope $5(\mathrm{C})$ and Isotope $6\left({ }^{1} \mathrm{H}\right)$, respectively. The dimensions and material composition of the polyethylene-reflected plutonium (PERP) metal sphere considered in this work are presented in Table A1 of Ref. [2]. For convenient reference, it is reproduced as below.

The PARTISN [7] computations of the neutron flux used the MENDF71X [9] 618-group cross section data collapsed to $G=30$ energy groups, as well as a $\mathrm{P}_{3}$ Legendre expansion of the scattering cross section and a fine-mesh spacing of $0.005 \mathrm{~cm}$ (comprising 759 meshes for the plutonium sphere of radius of 3.794 $\mathrm{cm}$, and 762 meshes for the polyethylene shell of thickness of $3.81 \mathrm{~cm}$ ). The firstand second-order response sensitivities were computed using an angular quadrature of $S_{256}$. The $3^{\text {rd }}$ - and $4^{\text {th }}$-order sensitivities of the leakage response with respect to the total cross sections were computed using an angular quadrature of $S_{32}$. The group boundaries of the $G=30$ energy groups are provided in the Appendix of Ref. [2]. The scattering and fission terms in Equation (1) contain implicitly a factor $1 / 4 \pi$, to conform to the convention used in PARTISN [7].

The quantities appearing in Equations (1) and (2) are defined as follows:

1) The quantity $\varphi^{g}(r, \boldsymbol{\Omega})$ is the customary "group-flux" for group

$g, g=1, \cdots, G$, and is the unknown state-function which is obtained by solving Equations (1) and (2).

2) The source $\boldsymbol{Q}^{g}(\boldsymbol{q})$ depends on the vector of model parameters $\boldsymbol{q}$, defined as follows:

$$
\boldsymbol{q} \triangleq\left[q_{1}, \cdots, q_{J Q}\right]^{\dagger} \triangleq\left[\lambda_{1}, \lambda_{2} ; F_{1}^{S F}, F_{2}^{S F} ; a_{1}, a_{2} ; b_{1}, b_{2} ; v_{1}^{S F}, v_{2}^{S F}\right]^{\dagger}, J Q=10 .
$$

Table A1. Dimensions and material composition of the PERP benchmark [2]

\begin{tabular}{|c|c|c|c|c|}
\hline Materials & Isotopes & $\begin{array}{l}\text { Weight } \\
\text { Fraction }\end{array}$ & $\begin{array}{l}\text { Density } \\
\left(\mathrm{g} / \mathrm{cm}^{3}\right)\end{array}$ & Zones \\
\hline $\begin{array}{c}\text { Material } 1 \\
\text { (plutonium metal) }\end{array}$ & $\begin{array}{l}\text { Isotope } 1\left({ }^{239} \mathrm{Pu}\right) \\
\text { Isotope } 2\left({ }^{240} \mathrm{Pu}\right) \\
\text { Isotope } 3\left({ }^{69} \mathrm{Ga}\right) \\
\text { Isotope } 4\left({ }^{71} \mathrm{Ga}\right)\end{array}$ & $\begin{array}{l}9.3804 \times 10^{-1} \\
5.9411 \times 10^{-2} \\
1.5152 \times 10^{-3} \\
1.0346 \times 10^{-3}\end{array}$ & 19.6 & $\begin{array}{l}\text { Homogeneous sphere of radius } \\
r_{1}=3.794 \mathrm{~cm} \text {, designated as } \\
\text { "material 1" and assigned to zone } \\
1\end{array}$ \\
\hline $\begin{array}{c}\text { Material } 2 \\
\text { (polyethylene) }\end{array}$ & Isotope $6\left({ }^{1} \mathrm{H}\right)$ & $1.4370 \times 10^{-1}$ & 0.95 & $\begin{array}{l}\text { Homogeneous spherical shell of } \\
\text { inner radius } r_{1}=3.794 \mathrm{~cm} \text { and } \\
\text { outer radius } r_{2}=7.604 \mathrm{~cm} \text {, } \\
\text { designated as "material } 2 \text { " and } \\
\text { assigned to zone } 2\end{array}$ \\
\hline
\end{tabular}


3) As indicated in Table A1, the PERP benchmark comprises 2 materials: "material 1" comprises 4 isotopes, numbered 1 through 4 , while "material 2" comprises 2 isotopes, numbered 5 and 6. In principle, PARTISN allows the same isotope to appear in different materials, in which case the atomic number density $N_{i, m}$ of an isotope $i$ in a material $m$ would be computed by using the formula $N_{i, m}=\rho_{m} w_{i, m} N_{A} / A_{i}$, where $\rho_{m}$ denotes the mass density of material $m$, $m=1,2, w_{i, m}$ denotes the weight fraction of isotope $i$ in material $m ; A_{i}$ denotes the atomic weight of isotope $i$, and $N_{A}$ denotes Avogadro's number. However, the two materials in the PERP benchmark contain only isotopes that are distinct from each other, so the subscript $m$ will not be needed if the formula for the atomic number density $N_{i}$ of an isotope $i, i=1, \cdots, I=6$, is interpreted as follows:

$$
N_{i} \triangleq \frac{\rho_{1} w_{i, 1} N_{A}}{A_{i}} \text {; for } i=1,2,3,4 ; N_{i} \triangleq \frac{\rho_{2} w_{i, 2} N_{A}}{A_{i}} \text {; for } i=5,6 \text {. }
$$

The atomic number densities $N_{i}, i=1, \cdots, I=6$ will be considered to be components of the vector $N$, defined below:

$$
N \triangleq\left[N_{1}, N_{2}, N_{3}, N_{4}, N_{5}, N_{6}\right]^{\dagger} \text {. }
$$

4) The scattering transfer cross section from energy group $g^{\prime}, g^{\prime}=1, \cdots, G$ into energy group $g, g=1, \cdots, G$ is denoted as $\Sigma_{s}^{g^{\prime} \rightarrow g}\left(\alpha ; r, \Omega^{\prime} \rightarrow \Omega\right)$ and is computed in terms of the $l$-th order Legendre coefficient $\sigma_{s, l, i}^{g^{\prime} \rightarrow g}$ using the following $3^{\text {rd }}$-order expansion in Legendre functions:

$$
\Sigma_{s}^{g^{\prime} \rightarrow g}\left(\alpha ; r, \mathbf{\Omega}^{\prime} \rightarrow \boldsymbol{\Omega}\right)=\sum_{i=1}^{I=6} N_{i} \sum_{l=0}^{I S C T=3}(2 l+1) \sigma_{s, l, i}^{g^{\prime} \rightarrow g} P_{l}\left(\mathbf{\Omega}^{\prime} \cdot \mathbf{\Omega}\right), g, g^{\prime}=1, \cdots, G,
$$

where ISCT $=3$ denotes the order of the expansion in Legendre polynomials. The microscopic scattering cross sections $\sigma_{s, l, i}^{g^{\prime} \rightarrow g}$ for isotope $i$, and from energy group $g^{\prime}$ into energy group $g$, are tabulated parameters. The zeroth-order (i.e., $l=0$ ) scattering cross sections must be considered separately from the higher order (i.e., $l \geq 1$ ) scattering cross sections, since the former contribute to the total cross sections (as noted below), while the latter do not. Aiming at reducing the proliferation of superscripts and subscripts when defining response sensitivities with respect to the microscopic scattering cross sections $\sigma_{s, l, i}^{g^{\prime} \rightarrow g}$, these cross sections will be considered to be components of a vector $\sigma_{s}$ defined below:

$$
\begin{aligned}
& \sigma_{s} \triangleq\left[s_{1}, \cdots, s_{J S X}\right]^{\dagger} \\
& \triangleq\left[\sigma_{s, l=0, i=1}^{g^{\prime}=1 \rightarrow g=1}, \sigma_{s, l=0, i=1}^{g^{\prime}=2 \rightarrow g=1}, \cdots, \sigma_{s, l=0, i=1}^{g^{\prime}=G \rightarrow g=1}, \sigma_{s, l=0, i=1}^{g^{\prime}=1 \rightarrow g=2}, \sigma_{s, l=0, i=1}^{g^{\prime}=2 \rightarrow g=2}, \cdots, \sigma_{s, l, i}^{g^{\prime} \rightarrow g}, \cdots, \sigma_{s, I S C T, i=I}^{G \rightarrow G}\right]^{\dagger}, \\
& l=0, \cdots, I S C T ; i=1, \cdots, I ; g, g^{\prime}=1, \cdots, G ; J S X=(G \times G) \times I \times(I S C T+1) .
\end{aligned}
$$

5) The total cross section for energy group $g, g=1, \cdots, G$ is denoted as $\Sigma_{t}^{g}(\boldsymbol{\alpha})$ and is computed using the following expression:

$$
\Sigma_{t}^{g}(\boldsymbol{\alpha})=\sum_{i=1}^{I} N_{i} \sigma_{t, i}^{g} ; \quad \sigma_{t, i}^{g}=\left[\sigma_{f, i}^{g}+\sigma_{c, i}^{g}+\sum_{g^{\prime}=1}^{G} \sigma_{s, l=0, i}^{g \rightarrow g^{\prime}}\right] .
$$

In Equation (94), the quantities $\sigma_{t, i}^{g}, \quad \sigma_{f, i}^{g}$ and $\sigma_{c, i}^{g}$ denote, respectively, the 
total microscopic cross section, the tabulated group microscopic fission, and the neutron capture cross sections for isotope $i$ and group $g$. Other nuclear reactions in the PERP benchmark are negligible. The total microscopic cross sections $\sigma_{t, i}^{g}$ involve three indices, which will proliferate exponentially when determining the higher-order (up to and including the $4^{\text {th }}$-order) sensitivities of the PERP leakage response with respect to these cross sections. In order to reduce as much as possible the proliferation of indices, it is useful to consider that the cross sections $\sigma_{t, i}^{g}$ are the components of a vector $t$, having $T \triangleq G \times I$ components and defined as follows:

$$
\begin{aligned}
& \boldsymbol{t} \triangleq\left[t_{1}, \cdots, t_{J T X}\right]^{\dagger} \triangleq\left[\sigma_{t, i=1}^{1}, \sigma_{t, i=1}^{2}, \cdots, \sigma_{t, i=1}^{G}, \cdots, \sigma_{t, i}^{g}, \cdots, \sigma_{t, i=I}^{1}, \cdots, \sigma_{t, i=I}^{G}\right]^{\dagger}, \\
& \quad \text { for } i=1, \cdots, I=6 ; g=1, \cdots, G=30 ; J T X \triangleq I \times G
\end{aligned}
$$

6) PARTISN [7] computes the quantity $\left(v \Sigma_{f}\right)^{g}(\alpha ; r)$ for each isotope $i$ and energy group $g$, as follows:

$$
\left(v \Sigma_{f}\right)^{g}(\alpha ; r)=\sum_{i=1}^{N F=2} N_{i} \sigma_{f, i}^{g} v_{i}^{g}, g=1, \cdots, G=30,
$$

where $\sigma_{f, i}^{g}$ denotes the microscopic fission cross section for isotope $i$ and energy group $g, v_{i}^{g}$ denotes the average number of neutrons per fission for isotope $i$ and energy group $g$, and $N F$ denotes the total number of fissionable isotopes.

$$
\begin{gathered}
\sigma_{f} \triangleq\left[\sigma_{f, i=1}^{1}, \sigma_{f, i=1}^{2}, \cdots, \sigma_{f, i=1}^{G}, \cdots, \sigma_{f, i}^{g}, \cdots, \sigma_{f, i=N F}^{1}, \cdots, \sigma_{f, i=N F}^{G}\right]^{\dagger} \triangleq\left[f_{1}, \cdots, f_{J F X}\right]^{\dagger}, \\
i=1, \cdots, N F ; g=1, \cdots, G ; J F X=G \times N F ; \\
\boldsymbol{v} \triangleq\left[v_{i=1}^{1}, v_{i=1}^{2}, \cdots, v_{i=1}^{G}, \cdots, v_{i}^{g}, \cdots, v_{i=N_{f}}^{1}, \cdots, v_{i=N F}^{G}\right]^{\dagger} \triangleq\left[f_{J F X+1}, \cdots, f_{J F X+J N U}\right]^{\dagger}, \\
\quad i=1, \cdots, N F ; g=1, \cdots, G ; J N U=G \times N F .
\end{gathered}
$$

7) The quantity $\chi^{g}(\alpha ; r)$ quantifies the fission spectrum in energy group $g$. The fission spectrum is considered to depend on the vector of parameters $\boldsymbol{p}$, defined as follows:

$$
\begin{aligned}
& \boldsymbol{p} \triangleq\left[p_{1}, \cdots, p_{J \chi}\right]^{\dagger} \triangleq\left[\chi_{i=1}^{g=1}, \chi_{i=1}^{g=2}, \cdots, \chi_{i=1}^{G}, \cdots, \chi_{i}^{g}, \cdots, \chi_{N F}^{G}\right]^{\dagger}, \\
& \quad \text { for } i=1, \cdots, N F ; g=1, \cdots, G ; \quad J \chi=G \times N F .
\end{aligned}
$$

8) In summary, the model parameters characterizing the PERP benchmark can all be considered to be the components of the following "vector of model parameters" denoted as $\alpha \triangleq\left[\alpha_{1}, \cdots, \alpha_{T P}\right]^{\dagger}$, where the subscript “ $T P$ ” stands for "Total number of model and response Parameters", and is defined below:

$$
\begin{aligned}
& \boldsymbol{\alpha} \triangleq\left[\alpha_{1}, \cdots, \alpha_{T P}\right]^{\dagger} \triangleq\left[\boldsymbol{c} ; \boldsymbol{N} ; \boldsymbol{\sigma}_{s} ; \boldsymbol{\sigma}_{t} ; \boldsymbol{\sigma}_{f} ; \boldsymbol{v} ; \boldsymbol{p}\right]^{\dagger}, \\
& \text { where } T P \triangleq J Q+I+J S X+J T X+J F X+J N U+J \chi
\end{aligned}
$$

9) The numerical model of the PERP benchmark contains 7477 parameters which have nonzero values and are subject to uncertainties, as follows: 1) 10 external neutron source parameters $\left.q_{j} ; 2\right) 6$ isotopic number densities $N_{i} ; 3$ ) 7101 group-averaged microscopic scattering cross sections $\sigma_{s, l, i}^{g^{\prime} \rightarrow g}$; 4) 180 grou- 
paveraged microscopic total cross sections $\sigma_{t, i}^{g}$;5) 60 group-averaged microscopic fission cross sections $\sigma_{f, i}^{g}$;6) 60 average-number of neutrons per fission $v_{i}^{g}$;7) 60 group-averaged fission spectrum parameters $\chi_{i}^{g}$. The vector $\boldsymbol{\alpha}$, which appears in the expression of the Boltzmann-operator $B^{g}(\boldsymbol{\alpha})$, represents the "vector of uncertain model parameters." The nominal values of the model parameters are denoted by using the superscript "zero", i.e., $\alpha^{0} \triangleq\left[\alpha_{1}^{0}, \cdots, \alpha_{T P}^{0}\right]^{\dagger}$.

\section{Nomenclature}

\section{Symbols}

$A$ : adjoint operator;

$A_{i}$ : atomic weight of isotope $i$;

$a_{k}, b_{k}$ : parameters used in Watt's fission spectra approximation for isotope $k$;

$B$ : forward operator;

$E^{g}$ : boundary of energy group $g$;

$F_{k}^{S F}$ : fraction of isotope $k$ decays that are spontaneous fission events;

$G$ : total number of energy groups;

$I$ : number of isotopes;

$J \chi$ : total number of parameters in vector $\boldsymbol{p}$;

$J Q$ : total number of parameters in vector $\boldsymbol{c}$;

$J F X$ : total number of parameters in vector $\sigma$;

$J S X$ : total number of parameters in vector $\sigma_{s}$;

$J T X$ : total number of parameters in vector $t$

$J N U$ : total number of parameters in vector $\boldsymbol{v}$;

$l$ : variable for the order of Legendre-expansion of the microscopic scattering cross sections, $l=1, \cdots, I S C T$;

$L, L(\alpha)$ : total neutron leakage from the PERP sphere; $m$ : materials;

$N_{A}$ : Avogadro's number;

$N_{f}$ : total number of fissionable isotopes;

$N_{i}$ : atomic number density for isotope $i$;

$P_{l}\left(\boldsymbol{\Omega}^{\prime} \cdot \boldsymbol{\Omega}\right)$ : spherical harmonics

$Q^{g}(r)$ : source term in group $g$,

$r$ : spatial (radial) variable;

$r_{d}$ : external radius of the PERP benchmark;

$S_{b}$ : outer surface of the PERP sphere;

$t_{j 1}, t_{j 2}$ : parameters in vector $\sigma_{t}$ indexed by $j 1$ and $j$;

$T P$ : total number of parameters in vector $\alpha$;

$w_{i, m}$ : weight fraction of isotope $i$ in material $m$;

Vectors and Matrices

$\alpha$ : vector of imprecisely known model parameters;

$\alpha^{0}$ : nominal values of the parameters in the vector $\alpha$;

$\boldsymbol{q}$ : vector of uncertain source parameters; 
$\boldsymbol{t}$ : vector of uncertain parameters characterizing the microscopic total cross sections;

$\sigma_{s}$ : vector of uncertain microscopic scattering cross sections;

$\sigma_{f}$ : vector of uncertain microscopic fission cross sections;

$\boldsymbol{v}$ : vector of uncertain average number of neutrons per fission;

$N$ : vector of uncertain atomic number densities;

$\boldsymbol{n}$ : the outward unit normal vector at each point on the sphere's outer boundary;

$\boldsymbol{p}$ : vector of uncertain fission spectrum parameters;

\section{Greek symbols}

$\alpha_{i}, \alpha_{j}:$ parameters in vector $\alpha$ indexed by $i$ and $j$;

$\delta$ : variations;

$\lambda_{k}$ : decay constant for isotope $k$,

$v_{i}^{g}$ : average number of neutrons produced per fission by isotope $i$ and group $g$

$v_{k}^{S F}$ : spontaneous emission of an average neutron of an isotope $k$

$\left(v \Sigma_{f}\right)^{g}$ : macroscopic fission cross section for energy group $g$;

$\rho_{m}:$ mass density of material $m$;

$\sigma$ : cross sections;

$\sigma_{c, i}^{g}$ : microscopic capture cross section in group $g$ of isotope $i$;

$\sigma_{f, i}^{g}:$ microscopic fission cross section in group $g$ of isotope $i$;

$\sigma_{s, l, i}^{g^{\prime} \rightarrow g}$ : the $l^{\text {th }}$ order Legendre-expanded microscopic scattering cross section from energy group $g^{\prime}$ into energy group $g$ for isotope $i$;

$\sigma_{t, i}^{g}:$ microscopic total cross section in group $g$ of isotope $i$;

$\Sigma_{t}^{g}$ : macroscopic total cross section for energy group $g$,

$\Sigma_{f}^{g}:$ macroscopic fission cross section for energy group $g$,

$\Sigma_{s}^{g^{\prime} \rightarrow g}\left(s ; \Omega^{\prime} \rightarrow \Omega\right)$ : macroscopic scattering transfer cross section from energy group $g^{\prime}$ into energy group $g$;

$\varphi^{g}(r, \boldsymbol{\Omega})$ : forward angular flux in group $g$ at point $r$ in direction $\Omega$;

$\chi^{g}$ : fission spectrum in energy group $g$,

$\psi^{(1), g}(r, \Omega)$ : adjoint angular flux in group $g$ at point $r$ in direction $\Omega$;

$\psi_{1}^{(2)}(g ; j, r, \Omega), \psi_{2}^{(2)}(g ; j ; r, \Omega): 2$ nd-level adjoint functions in group $g$ at point $r$ in direction $\Omega$ associated with the parameter indexed by $j\left(\right.$ e.g., $t_{j}$ );

$\boldsymbol{\Omega}, \boldsymbol{\Omega}^{\prime}$ : directional (solid angle) variables;

\section{Subscripts, superscripts}

f. fission;

$g, h$ : energy group variable $g, h=1, \cdots, G$;

$i$ : variable index;

$j$ : variable index;

$j 1, j 2, j 3, j 4$ : variable index;

$k$ : variable index;

$l$ : order of Legendre expansion associated with the microscopic scattering cross section; 
$m$ : index variable for materials, $m=1,2$;

t. total;

s. scattering;

Abbreviations

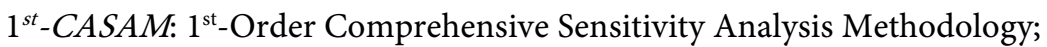

$1^{s t}-L A S S: 1^{\text {st }}$-Level Adjoint Sensitivity System;

$2^{\text {nd }}$-CASAM: $2^{\text {nd }}$-Order Comprehensive Sensitivity Analysis Methodology;

$2^{\text {nd }}-L A S S$ : $2^{\text {nd }}-$ Level Adjoint Sensitivity System;

$3^{\text {rd }}$-CASAM: $2^{\text {nd }}$-Order Comprehensive Sensitivity Analysis Methodology;

$3^{\text {rd }}$-LASS: $3^{\text {rd }}$-Level Adjoint Sensitivity System;

$4^{\text {th }}$-CASAM: $4^{\text {th }}$-Order Comprehensive Sensitivity Analysis Methodology;

$4^{\text {th }}$-LASS: $4^{\text {th }}$-Level Adjoint Sensitivity System;

$F D$ : finite-difference;

FSAM: Forward Sensitivity Analysis Methodology;

ISCT: order of the finite expansion in Legendre polynomial;

PERP: polyethylene-reflected plutonium. 